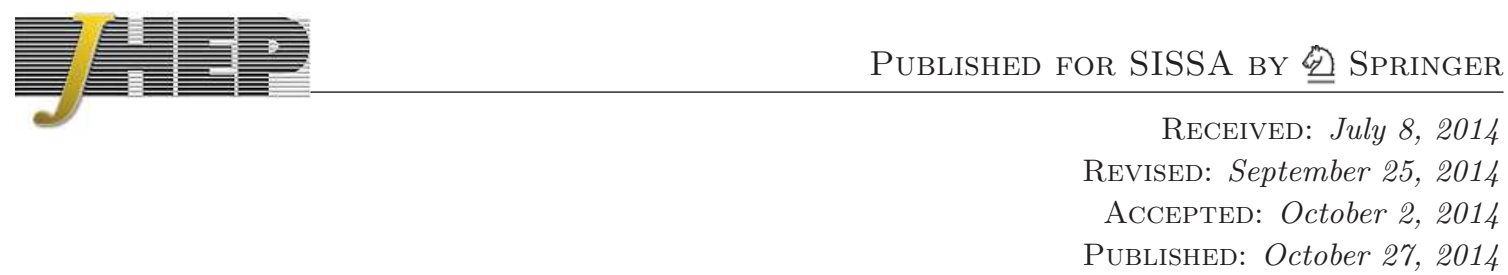

\title{
Notes on quantum entanglement of local operators
}

\author{
Masahiro Nozaki \\ Yukawa Institute for Theoretical Physics, Kyoto University, \\ Kitashirakawa Oiwakecho, Sakyo-ku, Kyoto 606-8502, Japan \\ E-mail: mnozaki@yukawa.kyoto-u.ac.jp
}

ABSTRACT: This is an expanded version of the short report arXiv:1401.0539, where we studied the time evolution of (Renyi) entanglement entropies for the excited state defined by acting a given local operator on the ground state. In the present paper, we introduce (Renyi) entanglement entropies of given local operators which are defined by late time values of excesses of (Renyi) entanglement entropies. They measure the degrees of freedom of local operators and characterize them in conformal field theories from the viewpoint of quantum entanglement. We explain how to compute them in free massless scalar field theories and we also investigate their time evolution. Our results can be interpreted in terms of the relativistic propagation of entangled pairs. The main new results which we acquire in the present paper are as follows. Firstly, we provide an explanation which shows that (Renyi) entanglement entropies of a specific operator are given by (Renyi) entanglement entropies whose reduced density matrices are given by the binomial distribution. That operator is constructed of only the scalar field. Secondly, we found the sum rule which (Renyi) entanglement entropies of those local operators obey. Those local operators are located separately. Moreover we argue that (Renyi) entanglement entropies of specific operators in conformal field theories are given by (Renyi) entanglement entropies whose reduced density matrices are given by the binomial distribution. These specific operators are constructed of single-species operators. We also argue that general operators obey the sum rule which we mentioned above.

Keywords: Conformal and W Symmetry, Field Theories in Lower Dimensions, Field Theories in Higher Dimensions

ARXIV EPRINT: 1405.5875

Open Access, (C) The Authors.

Article funded by $\mathrm{SCOAP}^{3}$.

doi:10.1007/JHEP10(2014)147 


\section{Contents}

1 Introduction $\quad 2$

2 The Rényi entanglement entropies for locally excited states and $\begin{array}{ll}\text { propagators } & 4\end{array}$

2.1 How to investigate the time evolution of (Rényi) entanglement entropies 4

2.1.1 An analytic continuation to real time 4

2.1.2 To compute (Rényi) entanglement entropies by using the replica method

2.2 Propagators on $\Sigma_{n}$

3 Results of Rényi entanglement entropies $\quad 9$

$\begin{array}{ll}3.1 \text { Results in 2-dimension } & 10\end{array}$

$\begin{array}{lll}3.1 .1 & \text { How to compute } \Delta S_{A}^{(n)} & 10\end{array}$

$\begin{array}{lll}3.1 .2 & \text { The derivatives of } \phi & 11\end{array}$

3.1.3 Exponential operators of $\phi \quad 11$

$\begin{array}{lll}3.2 & \text { Higher dimensional cases } & 12\end{array}$

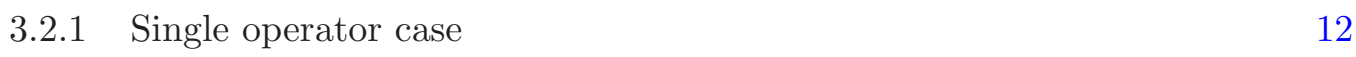

$\begin{array}{lll}3.3 & \text { Multiple operators case } & 14\end{array}$

$\begin{array}{lll}3.3 .1 & \text { Not simultaneous case } & 15\end{array}$

$\begin{array}{ll}3.3 .2 & \text { Simultaneous case } \\ & 17\end{array}$

4 Entangled pair interpretation $\quad 18$

4.1 A physical interpretation of $\Delta S_{A}^{(n)}$ in two dimensions 19

4.2 A physical interpretation in higher dimensions $(d>1) \quad 20$

$\begin{array}{lll}4.2 .1 & \text { Large } k \text { behavior } & 23\end{array}$

$\begin{array}{lll}\text { 4.2.2 } & \text { Generalization of } \Delta S_{A, j}^{(n) f} & 23\end{array}$

4.3 Sum rule of (Rényi) entanglement entropies of operators 23

$\begin{array}{ll}\text { 4.3.1 Generalized sum rule } & 24\end{array}$

5 General argument by using propagators $\quad \mathbf{2 5}$

$\begin{array}{lll}5.1 & \text { Properties of propagators } & 25\end{array}$

5.1.1 Time evolution of (Rényi) entanglement entropies for excited states 27

5.2 Sum rule of (Rényi) entanglement entropies of local operators 28

$\begin{array}{lll}5.3 \text { Generalization of sum rule } & 29\end{array}$

$\begin{array}{ll}5.4 \text { Conical singularity } & 30\end{array}$

$\begin{array}{lll}6 & \text { Discussions and conclusions } & 31\end{array}$ 


\section{Introduction}

Recently (Rényi) entanglement entropy has become a center of wide interest in a broad array of theoretical physics such as string theory, condensed matter physics and quantum information theory. It is a useful tool when we investigate the distinctive features of various quantum states. For example, it gives us the method of classifying the quantum structure of several states in condensed matter physics, e.g., [1-7]. The quantum entanglement is expected to be an important quantity which may shed light on the mechanism behind the AdS/CFT correspondence, e.g., [8-11]. In particular, the entanglement on the boundary is expected to be related to gravity in bulk e.g., [12-19]. Therefore, it is important to reveal the fundamental properties of (Rényi) entanglement entropy.

Remarkable properties of (Rényi) entanglement entropy have been obvious over the past a few years [20-35]. For example, a state is excited perturbatively so that the energy of its excitation is much smaller than the inverse of the subsystem size. If the subsystem size is small, its entanglement entropy is proportional to the energy included in it. Thus its entanglement entropy obeys a law analogous to the first law of thermodynamics. In the relativistic setup, its effective temperature is proportional to the inverse of the subsystem size. It does not depend on the parameters of strongly coupled gauge theories in large $N$ limit. In other words, it is universal in strongly coupled gauge theories in this limit.

In those works, we studied the property of the entanglement entropy when the subsystem size is very small. In this paper and the previous one [36], we define excited states by acting various local operators on the ground state. ${ }^{1}$ We study the time evolution of their (Rényi) entanglement entropies when its size is infinite. Their (Rényi) entanglement entropies show nontrivial time evolution. Their excesses finally approach certain finite values.

Properties of the excited state after quantum quenches have been studied more and are important. Before we explain how to compute (Rényi) entanglement entropies for locally excited states and our results, we would like to explain quantum quenches briefly. There are two classes of quenches; global quenches and local quenches. The global quenches are triggered by changing parameters homogeneously, e.g., [40-43]. When parameters are changed homogeneously, we are able to study the thermalization of a subsystem and its thermalization time by measuring its entanglement entropy. Their holographic duals are formation of a black hole, e.g., [44-58]. The local quenches are triggered by adding an interaction locally to a Hamiltonian or changing parameters locally in the Hamiltonian [59, 60]. When parameters are changed inhomogeneously, we can investigate the time evolution of the entanglement entropy locally. A falling particle in AdS spacetime is proposed as the holographic duals of these quenches, e.g., [23, 61].

In this paper, we consider excited states which are defined by acting a local operator on the ground state. The authors $[62,63]$ have studied the excited states similar to ours. It violates the time translational invariance and excites the ground state. In this setup, we

\footnotetext{
${ }^{1}$ We usually call excited states the eigenstates of a Hamiltonian which have energy higher than the energy of the ground state. In this paper we call them "standard" excited states. Their entanglement entropy has been studied in [37-39]. We call excited states or locally excited states states defined by acting local operators on the ground states. They are given by a linear combination of the eigenstates of Hamiltonian.
} 
can study the time evolution of their (Rényi) entanglement entropies. Therefore we can investigate how the entanglement propagates locally.

We would like to briefly explain how to compute (Rényi) entanglement entropies for these states and give an explanation of the results we obtain. In this work, we compute them in the free massless scalar field theory by the replica method. Here we choose the subsystem A to be a half of the total space. We define a locally excited state by acting a local operator : $\phi^{k}$ : on the ground state. We compute (Rényi) entanglement entropies for these states by using the replica method. After computing them, we perform an analytic continuation to real time and study their time evolution. We find that they finally approach certain finite constants. Their time evolution can be interpreted in terms of the relativistic propagation of entangled pairs.

In our previous paper [36], we derived (Rényi) entanglement entropies at late time for the state which is excited by $: \phi^{k}$ : from the entangled pair interpretation. In this paper, we introduce (Rényi) entanglement entropies of local operators which are defined by late time values of excesses of (Rényi) entanglement entropies. They measure the degrees of freedom of local operators and characterize them in conformal field theories from the viewpoint of quantum entanglement. They include (Rényi) entanglement entropies for an EPR state.

Finally we would like to summarize our new results. In the previous paper [36], we found that (Rényi) entanglement entropies of $: \phi^{k}$ : is given by (Rényi) entanglement entropies whose reduced density matrices are given by the binominal distribution only when the replica number and $k$ is small. In the present paper, we provide the explanation which shows that (Rényi) entanglement entropies of $: \phi^{k}:$ are given by those of the binomial distribution by using the replica method. The results which we obtain by using the replica method agree with those which we obtain in terms of entangled pairs.

We also obtain the sum rule for (Rényi) entanglement entropies for the states defined by acting the various operators on the ground state. They are given by the sum of (Rényi) entanglement entropies for the states defined by acting one of them on the ground state.

Moreover we argue that they can be generalized. We consider specific operators $\left(:\left(\partial^{m} \phi\right)^{k}:\right)$ which are composed of a single-species operator. Their (Rényi) entanglement entropies are given by (Rényi) entanglement entropies whose reduced density matrices are given by the binominal distribution. Those entropies of general operators obey the same sum rule as the one which we mentioned above.

This paper is organized as follows. In section 2, we compute (Rényi) entanglement entropies for the states defined by acting the various local operators on the ground state in the replica method. We also compute the Green function on the space which has a conical singularity. We explain an analytic continuation which is useful to investigate the time evolution of those entropies for excited states. In section 3, we calculate (Rényi) entanglement entropies for the states which excited by scalar operators and investigate their time evolution explicitly. In section 4, we interpret their time evolution in terms of entangled pair. We find that (Rényi) entanglement entropies of : $\phi^{k}:$ is given by those of binomial distribution. In section 5 , we explain the new results we obtain in this paper. We provide the explanation which shows that (Rényi) entanglement entropies of : $\phi^{k}:$ are given by those of binomial distribution by the replica method. We argue that those results can be generalized. In section 6 , we summarize the conclusions and discuss the future problems. 


\section{The Rényi entanglement entropies for locally excited states and propagators}

Let us study the time evolution of (Rényi) entanglement entropies for states generated by acting various local operators on the ground state. We will compute them by the replica methods. We will perform an analytic continuation to real time and investigate their real time evolution. In this section, we will explain the analytic continuation which is used to investigate their time evolution and also explain how to compute them. Those entropies are related to correlation functions of those operators as we explain later. In this paper, we compute them in the free massless scalar field theory in even dimensions. Therefore we compute Green functions on an $n$-sheeted geometry in the even dimensional free massless scalar field theory.

\subsection{How to investigate the time evolution of (Rényi) entanglement entropies}

\subsubsection{An analytic continuation to real time}

In this subsection, we would like to explain the analytic continuation which is used to investigate the real time evolution of (Rényi) entanglement entropies for states defined by acting various operators on the ground state. We consider a QFT in $d+1$ dimensional spacetime $\left(t, x^{i}\right)(i=1, \cdots, d)$. Let us define the locally excited state by

$$
\left|\Psi_{m}\right\rangle=\mathcal{N}^{-1} \mathcal{O}\left(-t_{1},-l, \mathbf{x}_{1}\right)|0\rangle
$$

where $\mathcal{O}$ is located at $\left(t=-t_{1}, x^{1}=-l, \mathbf{x}=\mathbf{x}_{1}\right)\left(\mathbf{x}=\left(x^{2}, x^{3}, \cdots, x^{d}\right)\right)$. Its density matrix is given by

$$
\hat{\rho}=\mathcal{N}^{-2} \mathcal{O}\left(-t_{1},-l, \mathbf{x}_{1}\right)|0\rangle\langle 0| \mathcal{O}^{\dagger}\left(-t_{1},-l, \mathbf{x}_{1}\right),
$$

where $\mathcal{N}$ is determined so as to $\operatorname{tr} \hat{\rho}=1$. The local operator $\mathcal{O}$ acts on the ground state at $t=-t_{1}$ and triggers the time evolution of quantum entanglement in the total system. We investigate its time evolution by measuring (Rényi) entanglement entropy at $t=0$.

To compute (Rényi) entanglement entropy for those locally excited states, we firstly introduce a regularization parameter $\epsilon$. By using it, $\hat{\rho}$ is deformed as follows,

$$
\hat{\rho}=\mathcal{N}^{-2} e^{-i H t_{1}} e^{-\epsilon H} \mathcal{O}\left(-l, \mathbf{x}_{1}\right)|0\rangle\langle 0| \mathcal{O}^{\dagger}\left(-l, \mathbf{x}_{1}\right) e^{-\epsilon H} e^{i H t_{1}} .
$$

High energy modes are suppressed by $e^{-\epsilon H}$. Thus its (Rényi) entanglement entropy is regulated.

We define a density matrix by

$\hat{\rho}=\mathcal{N}^{-2} e^{\tau_{e} H} \mathcal{O}\left(-l, \mathbf{x}_{1}\right)|0\rangle\left\langle 0\left|\mathcal{O}^{\dagger}\left(-l, \mathbf{x}_{1}\right) e^{-\tau_{l} H}=\mathcal{N}^{-2} \mathcal{O}\left(\tau_{e},-l, \mathbf{x}_{1}\right)\right| 0\right\rangle\langle 0| \mathcal{O}^{\dagger}\left(\tau_{l},-l, \mathbf{x}_{1}\right)$,

where $\tau_{e}=-\epsilon, \tau_{l}=\epsilon$ respectively. $\epsilon$ is the regularization parameter for the two point function of $\mathcal{O}$ and $\mathcal{O}^{\dagger}$.

After computing its (Rényi) entanglement entropies, we perform an analytic continuation to real time as follows,

$$
\begin{aligned}
\tau_{e} & =-\epsilon-i t_{1}, \\
\tau_{l} & =\epsilon-i t_{1} .
\end{aligned}
$$

The relation between the polar coordinate and $\epsilon, t_{1}$ is shown in figure 1 . Thus we investigate the evolution of $\Delta S_{A}^{(n)}$ for excited states given by (2.1) with $t_{1}$. 


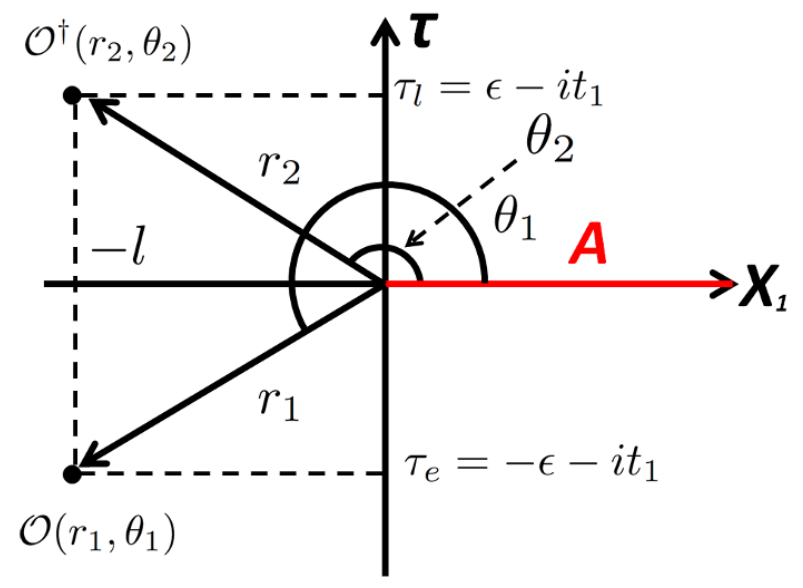

Figure 1. After the analytic continuation, $r_{1}, r_{2}, \theta_{1}$, and $\theta_{2}$ are related to $\epsilon, t_{1}$ and $l$ as in this figure.

\subsubsection{To compute (Rényi) entanglement entropies by using the replica method}

Let us compute (Rényi) entanglement entropies for the density matrix defined by (2.4). Local operators $\mathcal{O}$ and $\mathcal{O}^{\dagger}$ are located at $\left(\tau=\tau_{e}, x^{1}=-l, \mathbf{x}=\mathbf{x}_{1}\right)$ and $\left(\tau=\tau_{l}, x^{1}=\right.$ $\left.-l, \mathbf{x}=\mathbf{x}_{1}\right)$ respectively.

To define (Rényi) entanglement entropies, we choose a subsystem A to be a half space $x^{1} \geq 0 .^{2}$ Tracing out the degrees of freedom in the subsystem $\mathrm{B}$, we define a reduced density matrix by

$$
\hat{\rho}_{A}=\operatorname{tr}_{B} \hat{\rho} .
$$

Its $n$-th Rényi entanglement entropy is defined by

$$
S_{A}^{(n)}=\frac{1}{1-n} \log \operatorname{tr}_{A} \hat{\rho}_{A}^{n}
$$

We would like to study its excess. Therefore we subtract (Rényi) entanglement entropies for the ground state from those for the locally excited state and we define their excesses by

$$
\Delta S_{A}^{(n)}=\frac{1}{1-n} \log \left(\frac{\operatorname{tr} A \hat{\rho}_{A}^{n}}{\operatorname{tr}_{A} \hat{\rho}_{0 A}^{n}}\right) .
$$

$\hat{\rho}_{0}$ is a normalized reduced density matrix for the ground state.

Let us compute $\Delta S_{A}^{(n)}$ by the path-integral as in [65-67]. It is useful to introduce a polar coordinate. The location of operators is shown in figure 1 .

The wave functional $\Psi\left(\Phi_{0}\left(x^{i}\right)\right)$ for this state are given by path-integrating from $\tau=$ $-\infty$ to $\tau=0 . \Psi\left(\Phi_{0}\left(x^{i}\right)\right)$ is given by

$$
\Psi\left(\Phi_{0}\left(x^{i}\right)\right)=\int_{\Phi\left(\tau=-\infty, x^{i}\right)}^{\Phi\left(\tau=0, x^{i}\right)=\Phi_{0}\left(x^{i}\right)} D \Phi \mathcal{O}\left(r_{1}, \theta_{1}\right) e^{-S[\Phi]},
$$

\footnotetext{
${ }^{2}$ Of course, we are able to choose any shape as the subsystem A. We will compute (Rényi) entanglement entropy for the region $x^{1} \geq 0$ later. Therefore we choose subsystem A to be a half space of the total space.
} 
where $\Phi$ is general field of the theory. Then the components of the reduced matrix are given by,

$$
\begin{aligned}
& {\left[\hat{\rho}_{A}\right]_{\Phi_{1}\left(x^{i}\right) \Phi_{2}\left(x^{i}\right)}} \\
& =\left(Z_{1}^{\mathrm{EX}}\right)^{-1} \int_{\Phi\left(-\infty, x^{i}\right)}^{\Phi\left(\infty, x^{i}\right)} D \Phi \mathcal{O}^{\dagger}\left(r_{2}, \theta_{2},\right) \mathcal{O}\left(r_{1}, \theta_{1}\right) e^{-S[\Phi]} \\
& \quad \times \delta\left(\Phi\left(-\delta, x^{i}\right)-\Phi_{1}\left(x^{i}\right)\right) \cdot \delta\left(\Phi\left(\delta, x^{i}\right)-\Phi_{2}\left(x^{i}\right)\right)
\end{aligned}
$$

where we introduce a lattice spacing $\delta(\ll 1)$. $Z_{1}^{\mathrm{EX}}$ is defined by

$$
Z_{1}^{\mathrm{EX}}=\int_{\Phi\left(-\infty, x^{i}\right)}^{\Phi\left(\infty, x^{i}\right)} D \Phi \mathcal{O}^{\dagger}\left(r_{2}, \theta_{2}\right) \mathcal{O}\left(r_{1}, \theta_{1}\right) e^{-S(\Phi)} .
$$

Then $\operatorname{tr} \hat{\rho}_{A}^{n}$ is given by a partition function where $2 n$ operators are inserted,

$$
\begin{aligned}
\operatorname{tr}_{A} \hat{\rho}_{A}^{n}= & \frac{Z_{n}^{\mathrm{EX}}}{\left(Z_{1}^{\mathrm{EX}}\right)^{n}} \\
= & \left(Z_{1}^{\mathrm{EX}}\right)^{-n} \int_{\Phi\left(-\infty, x^{i}\right)}^{\Phi\left(\infty, x^{i}\right)} D \Phi \mathcal{O}^{\dagger}\left(r_{2}, \theta_{2}\right) \mathcal{O}\left(r_{1}, \theta_{1}\right) \cdots \mathcal{O}^{\dagger}\left(r_{2}, \theta_{2, k}\right) \mathcal{O}\left(r_{1}, \theta_{1, k}\right) \\
& \cdots \mathcal{O}^{\dagger}\left(r_{2}, \theta_{2, n}\right) \mathcal{O}\left(r_{1}, \theta_{1, n}\right) e^{-S[\Phi]}
\end{aligned}
$$

where operators are periodically located on an $n$ sheeted geometry $\Sigma_{n}$ and $\theta_{i, k}=\theta_{i}+$ $2 \pi(k-1)(i=1,2, k=1 \cdots n) . \Sigma_{n}$ is constructed by gluing the subsystem A on a sheet to the subsystem A on the next sheet as in figure 2. The reduced density matrix for the ground state is given by

$$
\operatorname{tr}_{A} \hat{\rho}_{0 A}^{n}=\frac{Z_{n}}{Z_{1}}
$$

where $Z_{n}$ is the partition function on the $n$ sheeted geometry and $Z_{1}$ is the partition function on $R^{d+1}$.

Substituting (2.12) and (2.13) into (2.8), the path integral representation of $\Delta S_{A}^{(n)}$ is given by

$$
\begin{aligned}
\Delta S_{A}^{(n)}= & \frac{1}{1-n}\left(\log \frac{\operatorname{tr}{ }_{A} \rho_{A}^{n}}{\rho_{0 A}}-n \log \frac{\operatorname{tr} \rho}{\operatorname{tr} \rho_{0}}\right)=\frac{1}{1-n}\left(\log \frac{Z_{n}^{\mathrm{EX}}}{Z_{n}}-n \log \frac{Z_{1}^{\mathrm{EX}}}{Z_{1}}\right) \\
= & \frac{1}{1-n}\left(\log \left\langle\mathcal{O}^{\dagger}\left(r_{2}, \theta_{2, n}\right) \mathcal{O}\left(r_{1}, \theta_{1, n}\right) \cdots \mathcal{O}^{\dagger}\left(r_{2}, \theta_{2,1}\right) \mathcal{O}\left(r_{1}, \theta_{1,1}\right)\right\rangle_{\Sigma_{n}}\right. \\
& \left.-n \log \left\langle\mathcal{O}^{\dagger}\left(r_{2}, \theta_{2,1}\right) \mathcal{O}\left(r_{1}, \theta_{1}\right)\right\rangle_{\Sigma_{1}}\right) .
\end{aligned}
$$

The first term of the last line in (2.14) is given by a $2 n$ point function of $\mathcal{O}$ and $\mathcal{O}^{\dagger}$ on $\Sigma_{n}$. The second term in (2.14) is given by a 2 point function of $\mathcal{O}$ and $\mathcal{O}^{\dagger}$ on $\Sigma_{1}$. Here $\Sigma_{1}$ is usual $d+1$ dimensional Euclidean space. ${ }^{3}$

Here only one operator acts on the ground state. However the result in (2.14) can be generalized to that for the excited state defined by acting various operators on the ground state as we will explain later.

\footnotetext{
${ }^{3}$ The authors in $[39,64]$ has found this relation between the excess of Rényi entanglement entropy and the correlation functions of operators.
} 


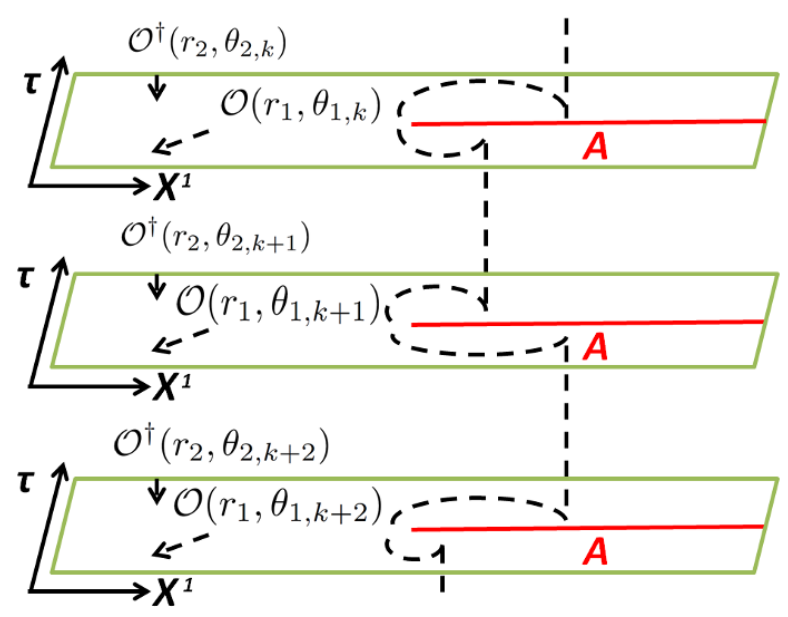

Figure 2. An $n$ sheeted geometry $\Sigma_{n}$ is constructed by gluing the subsystem A on a sheet to the subsystem $A$ on the next sheet. Fields acquire the phase shift of $2 \pi$ if they go around the boundary of A on the each sheet of $\Sigma_{n}$.

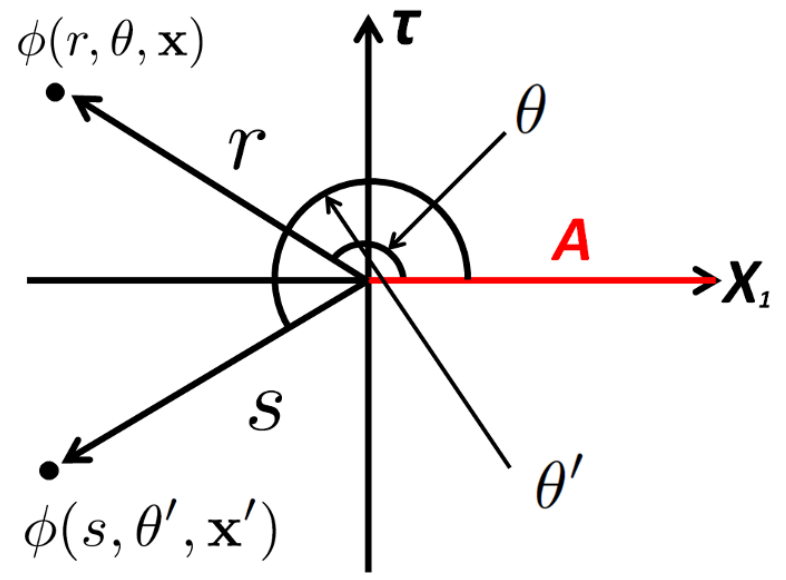

Figure 3. The subsystem $\mathrm{A}$ is given by the region $x_{1} \geq 0, \tau=0$. Operators are located at $(r, \theta, \mathbf{x}),\left(s, \theta^{\prime}, \mathbf{x}^{\prime}\right)$ respectively. $\mathbf{x}$ are the coordinates along the direction vertical to the plane which has a conical singularity.

\subsection{Propagators on $\Sigma_{n}$}

In the previous subsection, we defined excesses of (Rényi) entanglement entropies for locally excited states in general field theories. From now on, we will explain how to compute them and their time evolution in the free massless scalar field theory. $\Delta S_{A}^{(n)}$ are related to the $2 n$ point function on $\Sigma_{n}$ and the 2 point function on $\Sigma_{1}$ as in (2.14). Therefore we explain how to compute the Green function of $\phi$ on the even dimensional $\Sigma_{n}{ }^{4}$

We choose the region of the subsystem $\mathrm{A}$ to be a half $\left(x^{1} \geq 0\right)$ of the total space as in figure 3. We introduce a polar coordinate as $x^{1}+i \tau=r e^{i \theta}(0 \leq r<\infty, 0 \leq \theta<2 n \pi)$.

\footnotetext{
${ }^{4}$ It is technically difficult to compute the Green function of $\phi$ on the odd dimensional $\Sigma_{n}$. Therefore, we explain how to compute it on the even dimensional $\Sigma_{n}$.
} 
The Green function on $\Sigma_{n}$ is defined by

$$
\mathcal{L} G\left(r, s, \theta, \theta^{\prime}, \mathbf{x}, \mathbf{x}^{\prime}\right)=\left(\partial_{r}^{2}+\frac{1}{r} \partial_{r}+\frac{1}{r^{2}} \partial_{\theta}^{2}+\partial_{\mathbf{x}}^{2}\right) G\left(r, s, \theta_{a}, \theta_{b}, \mathbf{x}, \mathbf{x}^{\prime}\right)=-\delta\left(x-x^{\prime}\right),
$$

where $\mathbf{x}=\left(x^{2}, x^{3}, \cdots, x^{d}\right)$ and $x=\left(\tau, x^{1}, x^{2}, x^{3}, \cdots, x^{d}\right)$. Then we can expand it in terms of eingenfunctions $v(r, \theta, \mathbf{x})$,

$$
\mathcal{L} v(r, \theta, \mathbf{x})=\lambda v(r, \theta, \mathbf{x}),
$$

where $v(r, \theta, \mathbf{x})=v(r, \theta+2 n \pi, \mathbf{x})$. As in [68], it is given by

$$
G\left(r, s, \theta, \theta^{\prime}, \mathbf{x}, \mathbf{x}^{\prime}\right)=\frac{1}{2 \pi n} \sum_{l=0}^{\infty} d_{l} \int_{0}^{\infty} d k \int \frac{d k_{\perp}^{d-1}}{(2 \pi)^{d-1}} \frac{k \cdot J_{\frac{l}{n}}(k r) J_{\frac{l}{n}}(k s)}{k^{2}+k_{\perp}^{2}} e^{i \mathbf{k}_{\perp} \cdot\left(\mathbf{x}^{\prime}-\mathbf{x}\right)} \cos \left(\frac{\theta-\theta^{\prime}}{n} l\right)
$$

where $d_{0}=1, d_{l>0}=2$ and $J_{\nu}(x)$ is the Bessel function of the first kind.

To integrate with respect to $k_{\perp}$ in the right hand side of the equation in (2.17), we express it in terms of a Schwinger parameter. After that, its denominator is given by

$$
\frac{1}{k^{2}+k_{\perp}^{2}}=\int_{0}^{\infty} d u e^{-\left(k^{2}+k_{\perp}^{2}\right) u}
$$

After expressing it in terms of the Schwinger parameter, we integrate with respect to $k_{\perp}$ in the right hand side of the equation in (2.17),

$$
\int \frac{d k_{\perp}^{d-1}}{(2 \pi)^{d-1}} e^{-u\left(k_{\perp}\right)^{2}+i \mathbf{k}_{\perp} \cdot\left(\mathbf{x}^{\prime}-\mathbf{x}\right)}=\frac{1}{(2 \sqrt{u \pi})^{d-1}} e^{-\frac{1}{4 u}\left(\mathbf{x}^{\prime}-\mathbf{x}\right)^{2}} .
$$

Moreover, we integrate with respect to $k$ in (2.17). If we use the formula of the Bessel function, the integral of $k$ from 0 to $\infty$ in (2.17) can be performed,

$$
\int_{0}^{\infty} d k k e^{-u k^{2}} J_{\frac{l}{n}}(k r) J_{\frac{l}{n}}(k s)=\frac{1}{2 u} e^{-\frac{r^{2}+s^{2}}{4 u}} I_{\frac{l}{n}}\left(\frac{r s}{2 u}\right)
$$

where $I_{\frac{l}{n}}(x)$ is the modified Bessel function of the first kind. $I_{\frac{l}{n}}(x)$ is given by

$$
I_{\frac{l}{n}}\left(\frac{r s}{2 u}\right)=\frac{1}{2 \pi i} \int_{\infty-i \pi}^{\infty+i \pi} d t e^{\frac{r s}{2 u} \cosh t-\frac{l}{n} t}
$$

where the contour in the complex plane is shown in figure 4.

After performing the integral of $u, G\left(r, s, \theta, \theta^{\prime}, \mathbf{x}, \mathbf{x}^{\prime}\right)$ is given by

$$
\begin{aligned}
G\left(r, s, \theta, \theta^{\prime}, \mathbf{x}, \mathbf{x}^{\prime}\right)= & \frac{\Gamma\left(\frac{d-1}{2}\right)}{4 n \pi\left(2 \pi^{\frac{1}{2}}\right)^{d-1}} \\
& \times \frac{1}{2 i \pi} \sum_{l=0}^{\infty} d_{l} \int_{\infty-i \pi}^{\infty+i \pi} d t \frac{4^{\frac{d-1}{2}} e^{-\frac{l}{n} t} \cdot \cos \left(\frac{l\left(\theta-\theta^{\prime}\right)}{n}\right)}{\left\{\left(\left(\mathbf{x}^{\prime}-\mathbf{x}\right)^{2}+r^{2}+s^{2}-2 r s \cosh t\right)\right\}^{\frac{d-1}{2}}}
\end{aligned}
$$

When $d$ is odd, we can perform the integral of $t$. 


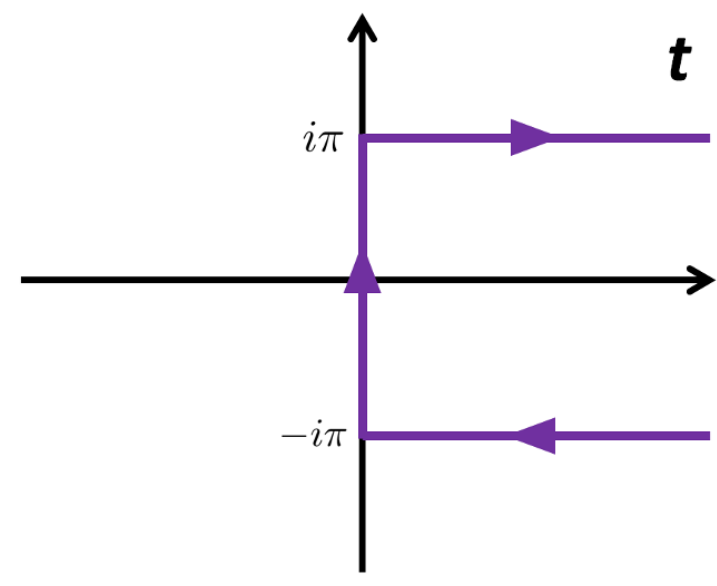

Figure 4. The purple line corresponds to the integral contour in the complex plane.

Below we will mainly investigate the time evolution of (Rényi) entanglement entropy for excited states in 4 and 6 dimensional cases. To evaluate (2.14), we would like to give the explicit form of the Green function on 4 and 6 dimensional $\Sigma_{n}$. When $d$ is 3 , $G\left(r, s, \theta, \theta^{\prime}, \mathbf{x}, \mathbf{x}^{\prime}\right)$ is given by

$$
G\left(r, s, \theta, \theta^{\prime}, \mathbf{x}, \mathbf{x}^{\prime}\right)=\frac{1}{4 n \pi^{2} r s\left(a-a^{-1}\right)} \frac{a^{\frac{1}{n}}-a^{-\frac{1}{n}}}{a^{\frac{1}{n}}+a^{-\frac{1}{n}}-2 \cos \left(\frac{\theta-\theta^{\prime}}{n}\right)},
$$

where the parameter $a$ is given by

$$
\frac{a}{1+a^{2}}=\frac{r s}{\left|\mathbf{x}^{\prime}-\mathbf{x}\right|^{2}+r^{2}+s^{2}}
$$

as in [6, 69-71]. When $d$ is $5, G\left(r, s, \theta, \theta^{\prime}, \mathbf{x}, \mathbf{x}^{\prime}\right)$ is given by

$$
\begin{aligned}
G\left(r, s, \theta, \theta^{\prime}, \mathbf{x}, \mathbf{x}^{\prime}\right) \\
=\frac{1}{4 \pi^{3} n^{2}(r s)^{2}\left(a-a^{-1}\right)^{2}} \\
\quad \times\left[\frac{2\left(\left(a^{\frac{1}{n}}+a^{-\frac{1}{n}}\right) \cos \left(\frac{\theta-\theta^{\prime}}{n}\right)-2\right)}{\left(a^{\frac{1}{n}}+a^{-\frac{1}{n}}-2 \cos \left(\frac{\theta-\theta^{\prime}}{n}\right)\right)^{2}}+\frac{n\left(a+a^{-1}\right)\left(a^{\frac{1}{n}}-a^{-\frac{1}{n}}\right)}{\left(a^{\frac{1}{n}}-a^{-\frac{1}{n}}-2 \cos \left(\frac{\theta-\theta^{\prime}}{n}\right)\right)\left(a-a^{-1}\right)}\right] .
\end{aligned}
$$

By using these Green functions, we will study the time evolution of (Rényi) entanglement entropies for locally excited states.

\section{Results of Rényi entanglement entropies}

Let us study the time evolution of (Rényi) entanglement entropies for various locally excited states in 2, 4 and 6 dimensional free massless scalar field theories. They will approach finite constant values at late time. Here, we will explain how to compute them. 
First, we will study them in two dimensional examples. In those examples, we can compute them by using a conformal map. We will explain results of $\Delta S_{A}^{(2)}$ for states defined by acting several operators on the ground state. We choose $\partial \phi,: \partial \phi \partial \phi:,: \bar{\partial} \phi \partial \phi$, $: e^{ \pm i \alpha \phi}:$ and $: e^{i \alpha \phi}:+c: e^{-i \alpha \phi}:$ as them ( $c$ is a complex number.). We define excited states by acting them on the ground state. $\Delta S_{A}^{(2)}$ for only the last one shows the nontrivial time evolution. We assume they are located at $\left(t=-t_{1}, x^{1}=-l\right)$.

In 4 and 6 dimensions, we define excited states by acting : $\phi^{k}$ : on the ground state. $\Delta S_{A}^{(n)}$ for them show the similar time evolution and approach finite constants at late time in both dimensions. $\Delta S_{A}^{(n)}$ in 6 dimensions increase milder than those in 4 dimensions. However the final values of $\Delta S_{A}^{(n)}$ do not depend on spacetime dimension but depend on $k$ and $n$. Therefore we would like to explain how to compute them in only 4 dimensions.

Here we compute them in two illustrative examples. In the first example, we compute $\Delta S_{A}^{(2)}$ for the state which is excited by the local operator $\phi$. We also compute that for the state which is excited by two local operators $\phi \phi$ in the second example. In the latter example, we assume two operators are located separately and we would like to study the time evolution of $\Delta S_{A}^{(2)}$ in two setups.

In the first setup, one operator $\phi$ is located at $\left(t=-t_{1}, x^{1}=-L\right)$ and another one is located at $\left(t=-t_{2}, x^{1}=-l\right)$ where $l \neq L, t_{1} \geq L$. If $t_{2} \leq l, \Delta S_{A}^{(2)}$ is given by a finite constant which depends on the parameters $t_{1}, L$. If $t_{2} \geq l, \Delta S_{A}^{(2)}$ shows the nontrivial time evolution and finally approaches a certain constant value. It also depends on only two parameters $t_{1}, L$. In both cases, $\Delta S_{A}^{(2)}$ is given by the sum of (Rényi) entanglement entropy for the state which is excited by $\phi .^{5}$

\subsection{Results in 2-dimension}

In this subsection, we explain how to compute $\Delta S_{A}^{(n)}$ for locally excited states and also explain the results in two dimensional examples. We compute $\Delta S_{A}^{(n)}$ for states defined by acting derivatives of $\phi$ and exponentials of $\phi$ on the ground state. We choose $\partial \phi,: \partial \phi \partial \phi$ : and : $\bar{\partial} \phi \partial \phi:$ as derivatives of $\phi .: e^{ \pm i \alpha \phi}:$ and $: e^{i \alpha \phi}:+c: e^{-i \alpha \phi}:$ are chosen as its exponentials. ${ }^{6}$

\subsubsection{How to compute $\Delta S_{A}^{(n)}$}

In the two dimensional free massless scalar field theory, we can calculate $\Delta S_{A}^{(n)}$ by using a conformal map $\omega=z^{n}$. In this case, we do not need the Green function which we obtain in section 2. Here $z$ is the coordinate of $n$-sheeted geometry and $\omega$ is the coordinate of the flat plane. To compute derivatives and exponentials of $\phi$, we just have to obtain the two point function of $\phi$. The green function on the flat plane $\left(=\Sigma_{1}\right)$ is given by

$$
\left\langle\phi\left(\omega_{1}, \bar{\omega}_{1}\right) \phi\left(\omega_{2}, \bar{\omega}_{2}\right)\right\rangle=-\frac{1}{2} \log \left|\omega_{1}-\omega_{2}\right|^{2} .
$$

\footnotetext{
${ }^{5}$ In every case, we assume $l \geq 0$ and $L \geq 0$ for simplicity.

${ }^{6}$ In two and higher dimensions, we will study the time evolution of $\Delta S_{A}^{(n)}$ for states defined by acting local operators whose conformal dimensions are positive on the ground state. In two dimensions the conformal dimension of $\phi$ vanishes. It does not seem to be a local operator from the viewpoint of quantum entanglement. Therefore we will not explain $\Delta S_{A}^{(n)}$ for the state which excited by $\phi$ in this paper.
} 
We impose that the behavior of the two point function on $\Sigma_{n}$ is the same as the behavior of the Green function on $\Sigma_{1}$ if we take the limit $z_{1} \rightarrow z_{2}$. We also impose that this two point function on $\Sigma_{n}$ satisfies the properties of Green function. By using the conformal map $\omega=z^{n}$, two point function of $\phi$ on $\Sigma_{n}$ is given by

$$
\left\langle\phi\left(z_{1}, \overline{z_{1}}\right) \phi\left(z_{2}, \bar{z}_{2}\right)\right\rangle_{\Sigma_{n}}=-\frac{1}{2} \log \left|z_{1}^{\frac{1}{n}}-z_{2}^{\frac{1}{n}}\right|+\frac{1}{2} \log \left(\frac{\left|z_{1}\right|^{\frac{1}{n}-1}}{n}\right)+\frac{1}{2} \log \left(\frac{\left|z_{2}\right|^{\frac{1}{n}-1}}{n}\right) .
$$

After substituting the propagators in (3.2) into (2.14), we perform the analytic continuation defined in (2.5) to real time. After that, we can study the time evolution of $\Delta S_{A}$ for states defined by acting local operators on the ground state.

\subsubsection{The derivatives of $\phi$}

Here we compute the $\Delta S_{A}^{(2)}$ for locally excited states defined by acting derivatives of $\phi$ on the ground state. We choose $\partial \phi(z, \bar{z}),: \partial \phi(z, \bar{z}) \partial \phi(z, \bar{z}):,: \bar{\partial} \phi(z, \bar{z}) \partial \phi(z, \bar{z}):$ as them.

After computing their second Rényi entanglement entropies, we perform the analytic continuation in (2.5) and take the limit $\epsilon \rightarrow 0$. In this limit, they vanish,

$$
\Delta S_{A}^{(2)}=0 .
$$

As we explain in the subsection 4.2 , these results can be physically interpreted in terms of the relativistic propagation of quasi-particles.

\subsubsection{Exponential operators of $\phi$}

We would like to explain the results of excited states generated by acting exponential operators of $\phi$ on the ground state such as : $e^{ \pm i \alpha \phi}:,: e^{i \alpha \phi}:+c: e^{-i \alpha \phi}: \Delta \Delta S_{A}^{(2)}$ for the states which are excited by $: e^{ \pm i \phi}:$ vanishes.

On the other hand, $\Delta S_{A}^{(2)}$ for the state defined by acting : $e^{i \alpha \phi}:+c: e^{-i \alpha \phi}:$ on the ground state does not vanish. In the replica method, $\Delta S_{A}^{(2)}$ for this state is given by

$$
\Delta S_{A}^{(2)}=-\log \left[\frac{\left\langle\mathcal{O}^{\dagger}\left(r_{2}, \theta_{2}+2 \pi\right) \mathcal{O}\left(r_{1}, \theta_{1}+2 \pi\right) \mathcal{O}^{\dagger}\left(r_{2}, \theta_{2}\right) \mathcal{O}\left(r_{1}, \theta_{1}\right)\right\rangle_{\Sigma_{2}}}{\left\langle\mathcal{O}^{\dagger}\left(r_{2}, \theta_{2}\right) \mathcal{O}\left(r_{1}, \theta_{1}\right)\right\rangle_{\Sigma_{1}}}\right],
$$

where $\mathcal{O}$ and $\mathcal{O}^{\dagger}$ are given by

$$
\begin{aligned}
\mathcal{O}\left(r_{1}, \theta_{1}\right) & =: e^{i \alpha \phi}:+c: e^{-i \alpha \phi}: \\
\mathcal{O}^{\dagger}\left(r_{1}, \theta_{1}\right) & =: e^{-i \alpha \phi}:+c^{*}: e^{i \alpha \phi}: .
\end{aligned}
$$

By substituting propagators in (3.2) into (3.4), we perform the analytic continuation in (2.5) and take the limit $\epsilon \rightarrow 0$. The time evolution of $\Delta S_{A}^{(2)}$ is given by the step function,

$$
\Delta S_{A}^{(2)}= \begin{cases}0 & t_{1} \leq 0 \\ -\log \left[\frac{1+|c|^{4}}{\left(1+|c|^{2}\right)^{2}}\right] & t_{1}>0\end{cases}
$$




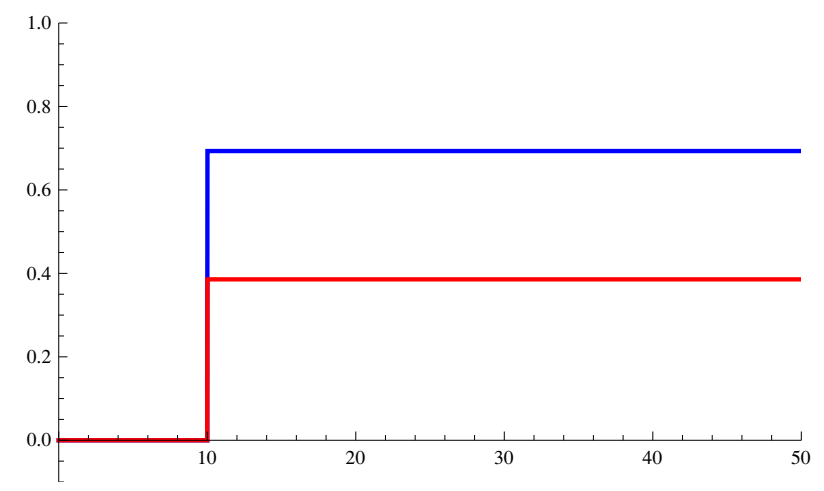

Figure 5. Plots of $\Delta S_{A}^{(2)}$ as functions of $t_{1}$ in the limit $\epsilon \rightarrow 0$. The vertical line corresponds to $\Delta S_{A}^{(2)}$. The horizontal line corresponds to $t_{1}$. Here we chose $l=10$. The red curve corresponds to the plot of $\Delta S_{A}^{(2)}$ for $|c|=\frac{1}{2}$. The blue curve corresponds to the plot of $\Delta S_{A}^{(2)}$ for $|c|=1$.

$\Delta S_{A}^{(2)}$ does not depend on the conformal dimension of the operator $\mathcal{O}$. This final value depends on only the complex constant $c$. When $|c|$ is 1 , the final value of $\Delta S_{A}^{(2)}$ is given by its maximum value. It is equivalent to second Rényi entanglement entropy for an EPR state. The time evolution of $\Delta S_{A}^{(2)}$ is plotted in figure 5. In the subsection 4.1, we interpret this result in terms of entangled quanta.

\subsection{Higher dimensional cases}

In this subsection, we compute $\Delta S_{A}^{(2)}$ and study its time evolution in two examples. In both cases, we compute $\Delta S_{A}^{(2)}$ for locally excited states by the replica method. After computing it, we perform the analytic continuation defined by (2.5) to real time and we study its real time evolution.

\subsubsection{Single operator case}

As we mentioned above, we compute $\Delta S_{A}^{(2)}$ for the excited state defined by acting a local oprerator $\phi$ in the 4 dimensional free massless scalar field theory. Let us calculate its second Rényi entanglement entropy and study its time evolution. We assume that $\phi$ is located at $\left(t=-t_{1}, x^{1}=-l\right)$. This locally excited state is given by

$$
|\Psi\rangle=\mathcal{N}^{-1} \phi\left(-t_{1},-l\right)|0\rangle
$$

We assume that $\phi$ is located at $\tau=\tau_{e}, x^{1}=-l$ in Euclidean space. A state defined by acting it on the ground state is given by

$$
|\Psi\rangle=\mathcal{N}^{-1} \phi\left(\tau_{e},-l\right)|0\rangle
$$

After mapping $\left(\tau, x^{1}\right)$ to $(r, \theta)$ as in figure $6, \Delta S_{A}^{(2)}$ for this state is given by

$$
\Delta S_{A}^{(2)}=-\log \left[\frac{\left\langle\phi\left(r_{1}, \theta_{1}\right) \phi\left(r_{2}, \theta_{2}\right) \phi\left(r_{1}, \theta_{1}+2 \pi\right) \phi\left(r_{2}, \theta_{2}+2 \pi\right)\right\rangle_{\Sigma_{2}}}{\left\langle\phi\left(r_{1}, \theta_{1}\right) \phi\left(r_{2}, \theta_{2}\right)\right\rangle_{\Sigma_{1}}^{2}}\right],
$$

where $\phi\left(r_{2}, \theta_{2}\right)$ is located at $\left(\tau=\tau_{l}, x^{1}=-l\right)$ as in figure 6 . 


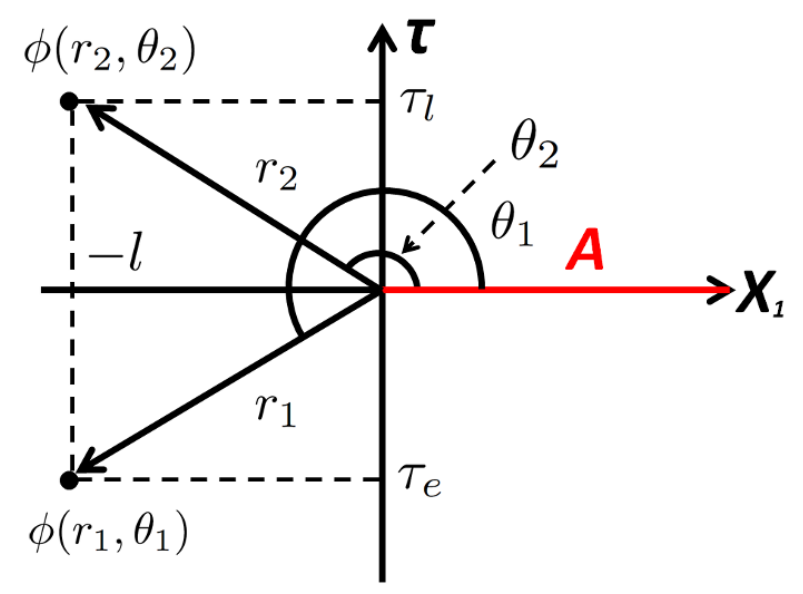

Figure 6. The location of operators.

To compute $\Delta S_{A}^{(2)}$, we need to compute the 4 point correlation function of $\phi$ on $\Sigma_{2}$ and the 2 point correlation function of $\phi$ on $\Sigma_{1}$. The Green functions on $\Sigma_{2}$ and $\Sigma_{1}$ are respectively given by

$$
\begin{aligned}
\left\langle\phi(r, \theta) \phi\left(s, \theta^{\prime}\right)\right\rangle_{\Sigma_{2}} & =\frac{1}{8 \pi^{2}(r+s)\left(r+s-2 \sqrt{r s} \cos \left(\frac{\theta-\theta^{\prime}}{2}\right)\right)}, \\
\left\langle\phi(r, \theta) \phi\left(s, \theta^{\prime}\right)\right\rangle_{\Sigma_{1}} & =\frac{1}{4 \pi^{2}\left(r^{2}+s^{2}-2 r s \cos \left(\theta-\theta^{\prime}\right)\right)} .
\end{aligned}
$$

By substituting them in (3.10) into (3.9), we perform the analytic continuation in (2.5) to real time.

In this analytic continuation, we can obtain following useful relations,

$$
\begin{aligned}
r_{1}^{2} & =l^{2}+\epsilon^{2}-t_{1}^{2}+2 i \epsilon t_{1}, \\
r_{2}^{2} & =l^{2}+\epsilon^{2}-t_{1}^{2}-2 i \epsilon t_{1}, \\
r_{1} r_{2} \cos \left(\theta_{1}-\theta_{2}\right) & =l^{2}-\epsilon^{2}-t_{1}^{2} .
\end{aligned}
$$

After performing this analytic continuation, we take the limit $\epsilon \rightarrow 0$. In this limit, the two point function of $\phi$ on $\Sigma_{1}$ is given by

$$
\left\langle\phi\left(r_{1}, \theta_{1}\right) \phi\left(r_{2}, \theta_{2}\right)\right\rangle_{\Sigma_{1}}=\frac{1}{16 \pi^{2} \epsilon^{2}} .
$$

The leading term of its four point function on $\Sigma_{2}$ is $\mathcal{O}\left(\epsilon^{-4}\right)$ in this limit. Only specific propagators can contribute to it. We call those propagators dominant propagators. In the region $t_{1}<l$, dominant propagators are given by

$$
\left\langle\phi\left(r_{1}, \theta_{1}\right) \phi\left(r_{2}, \theta_{2}\right)\right\rangle_{\Sigma_{2}}=\left\langle\phi\left(r_{1}, \theta_{1}+2 \pi\right) \phi\left(r_{2}, \theta_{2}+2 \pi\right)\right\rangle_{\Sigma_{2}}=\frac{1}{16 \pi^{2} \epsilon^{2}}+\mathcal{O}\left(\epsilon^{0}\right) .
$$

In the region $t_{1} \geq l$, dominant propagators are given

$$
\begin{aligned}
\left\langle\phi\left(r_{1}, \theta_{1}\right) \phi\left(r_{2}, \theta_{2}\right)\right\rangle_{\Sigma_{2}} & =\left\langle\phi\left(r_{1}, \theta_{1}+2 \pi\right) \phi\left(r_{2}, \theta_{2}+2 \pi\right)\right\rangle_{\Sigma_{2}}=\frac{l+t_{1}}{32 \pi^{2} t_{1} \epsilon^{2}}+\mathcal{O}\left(\epsilon^{0}\right), \\
\left\langle\phi\left(r_{1}, \theta_{1}+2 \pi\right) \phi\left(r_{2}, \theta_{2}\right)\right\rangle_{\Sigma_{2}} & =\left\langle\phi\left(r_{1}, \theta_{1}\right) \phi\left(r_{2}, \theta_{2}+2 \pi\right)\right\rangle_{\Sigma_{2}}=\frac{-l+t_{1}}{32 \pi^{2} t_{1} \epsilon^{2}}+\mathcal{O}\left(\epsilon^{0}\right) .
\end{aligned}
$$




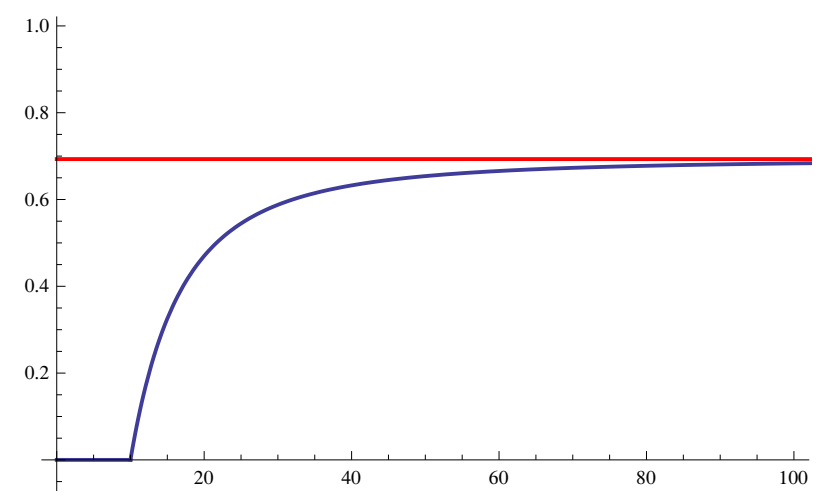

Figure 7. The evolution of $\Delta S_{A}^{(2)}$ with $t_{1}$ in the limit $\epsilon \rightarrow 0$. The vertical axis corresponds to $\Delta S_{A}^{(2)}$. The horizontal axis corresponds to $t_{1}$. Here we chose $l=10$. The blue curve is the $\Delta S_{A}^{(2)}$ for $\phi|0\rangle$. The red curve is the final value of $\Delta S_{A}^{(2)}$. It is given by $\log 2$.

The other propagators are at most $\mathcal{O}\left(\epsilon^{0}\right)$ in both regions. Therefore we can ignore them in the limit $\epsilon \rightarrow 0$.

In the region $t_{1}<l$, the four point correlation function of $\phi$ on $\Sigma_{2}$ is given by

$$
\left\langle\phi\left(r_{1}, \theta_{1}\right) \phi\left(r_{2}, \theta_{2}\right) \phi\left(r_{1}, \theta_{1}+2 \pi\right) \phi\left(r_{2}, \theta+2 \pi\right)\right\rangle_{\Sigma_{2}}=\frac{1}{256 \pi^{4} \epsilon^{4}}+\mathcal{O}\left(\epsilon^{-3}\right) .
$$

In the region $t_{1} \geq l$, it is given by

$$
\left\langle\phi\left(r_{1}, \theta_{1}\right) \phi\left(r_{2}, \theta_{2}\right) \phi\left(r_{1}, \theta_{1}+2 \pi\right) \phi\left(r_{2}, \theta_{2}+\pi\right)\right\rangle_{\Sigma_{2}}=\frac{l^{2}+t_{1}^{2}}{512 \pi^{4} t_{1}^{2} \epsilon^{4}}+\mathcal{O}\left(\epsilon^{-3}\right)
$$

In the limit $\epsilon \rightarrow 0$, second Rényi entanglement entropy for the excited state defined by acting $\phi$ on the ground state is given by

$$
\Delta S_{A}^{(2)}=\log \left[\frac{2 t_{1}^{2}}{l^{2}+t_{1}^{2}}\right]
$$

The time evolution of $\Delta S_{A}^{(2)}$ in (3.17) is plotted in figure 7 .

If we take the limit $t_{1} \rightarrow \infty$, its final value is given by

$$
\Delta S_{A}^{(2) f}=\log 2
$$

\subsection{Multiple operators case}

Let us compute second Rényi entanglement entropy $\Delta S_{A}^{(2)}$ for the state defined by acting two local operators $\phi \phi$ on the ground state. We assume they are located separately. We compute it in two different examples. In the first example, we act $\phi$ on the ground state at time $t=-t_{1}$ and act another one on it at time $t=-t_{2}$. We investigate the evolution of second Rényi entanglement entropy with $t_{2}$. In the second example, we act two operators on the ground state simultaneously. We also investigate the time evolution of second Rényi entanglement entropy. 


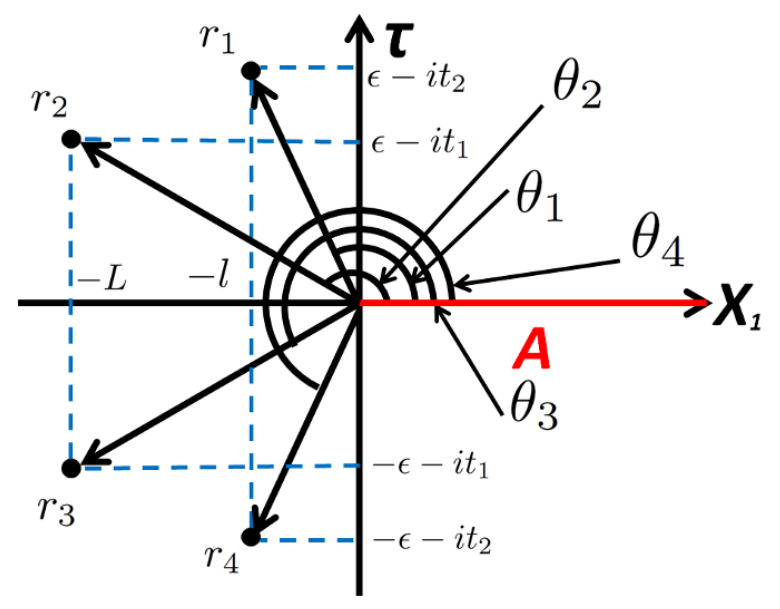

Figure 8. The relation between coordinates. $r, \theta$ are related to $l, L, t_{1}, t_{2}$ and $\epsilon$ after performing an analytic continuation to real time.

\subsubsection{Not simultaneous case}

Let us compute $\Delta S_{A}^{(2)}$ for the state which is excited by two local operators $\phi \phi$. One of them is located at $t=-t_{1}, x^{1}=-L$ as in figure 8. Here, we assume that $t_{1} \geq L$. If $t_{1} \leq L$, then the time evolution of $\Delta S_{A}^{(2)}$ is not affected by $\phi\left(-t_{1},-L\right)$. Another one is located at $t=-t_{2}, x^{1}=-l$. We would like to investigate the evolution of $\Delta S_{A}^{(2)}$ with $t_{2}$.

In this example, the excited state is given by

$$
|\Psi\rangle=\mathcal{N}^{-1} \mathcal{T} \phi\left(-t_{1},-L\right) \phi\left(-t_{2},-l\right)|0\rangle,
$$

where $\mathcal{T}$ is a time ordering operator. In the replica method, $\Delta S_{A}^{(2)}$ for it is given by

$\Delta S_{A}^{(2)}$

$=-\log \left[\frac{\left\langle\phi\left(r_{1}, \theta_{1}\right) \phi\left(r_{2}, \theta_{2}\right) \phi\left(r_{3}, \theta_{3}\right) \phi\left(r_{4}, \theta_{4}\right) \phi\left(r_{1}, \theta_{1}+2 \pi\right) \phi\left(r_{2}, \theta_{2}+2 \pi\right) \phi\left(r_{3}, \theta_{3}+2 \pi\right) \phi\left(r_{4}, \theta_{4}+2 \pi\right)\right\rangle_{\Sigma_{2}}}{\left\langle\phi\left(r_{1}, \theta_{1}\right) \phi\left(r_{2}, \theta_{2}\right) \phi\left(r_{3}, \theta_{3}\right) \phi\left(r_{4}, \theta_{4}\right)\right\rangle_{\Sigma_{1}}^{2}}\right]$.

To compute $\Delta S_{A}^{(2)}$, we have to compute the eight point correlation function of $\phi$ on $\Sigma_{2}$ and its four point function on $\Sigma_{1}$. Its Green functions are given by (3.10).

In Euclidean space, $\phi\left(-t_{1},-L\right)$ corresponds to $\phi\left(r_{2}, \theta_{2}\right)$ and $\phi\left(r_{3}, \theta_{3}\right)$. They are located at $\left(\tau=\tau_{l, 1}, x^{1}=-L\right)$ and $\left(\tau=\tau_{e, 1}, x^{1}=-L\right)$ respectively. On the other hand, $\phi\left(-t_{2},-l\right)$ corresponds to $\phi\left(r_{1}, \theta_{1}\right), \phi\left(r_{4}, \theta_{4}\right)$. They are located at $\left(\tau=\tau_{l, 2}, x^{1}=-l\right)$ and $\left(\tau=\tau_{e, 2}, x^{1}=-l\right)$ respectively as in figure 8. In addition to correlations in the case where one operator acts on the ground state, there are correlations which correspond to the correlation between $\phi\left(-t_{1},-l\right)$ and $\phi\left(-t_{2},-L\right)$ such as $\left\langle\phi\left(r_{1}, \theta_{1}\right) \phi\left(r_{2}, \theta_{2}\right)\right\rangle$, $\left\langle\phi\left(r_{1}, \theta_{1}\right) \phi\left(r_{2}, \theta_{2}+2 \pi\right)\right\rangle, \cdots$. It seems that we have to take their correlation functions into account. However they can not contribute to $\Delta S_{A}^{(2)}$ when we take the limit $\epsilon \rightarrow 0$. Therefore we can neglect their contribution. Below we will explain why these correlation functions can not contribute to $\Delta S_{A}^{(2)}$ in this limit. 
After substituting the propagators in (3.10) into (3.20), we perform an analytic continuation as follows,

$$
\begin{aligned}
\tau_{l, 1} & =\epsilon-i t_{1}, \\
\tau_{l, 2} & =\epsilon-i t_{2}, \\
\tau_{e, 1} & =-\epsilon-i t_{1}, \\
\tau_{e, 2} & =-\epsilon-i t_{2},
\end{aligned}
$$

as in figure 8. In this continuation, there are useful relations as follows,

$$
\begin{aligned}
r_{1}^{2} & =l^{2}+\epsilon^{2}-t_{2}^{2}-2 i \epsilon t_{2}, \\
r_{2}^{2} & =L^{2}+\epsilon^{2}-t_{1}^{2}-2 i \epsilon t_{1}, \\
r_{3}^{2} & =L^{2}+\epsilon^{2}-t_{1}^{2}+2 i \epsilon t_{1}, \\
r_{2}^{2} & =l^{2}+\epsilon^{2}-t_{2}^{2}+2 i \epsilon t_{2},
\end{aligned}
$$

and

$$
\begin{aligned}
& r_{1} r_{2} \cos \left(\theta_{1}-\theta_{2}\right)=l L+\epsilon^{2}-t_{1} t_{2}-i \epsilon\left(t_{1}+t_{2}\right), \\
& r_{1} r_{3} \cos \left(\theta_{1}-\theta_{3}\right)=l L-\epsilon^{2}-t_{1} t_{2}-i \epsilon\left(t_{1}-t_{2}\right), \\
& r_{1} r_{4} \cos \left(\theta_{1}-\theta_{4}\right)=l^{2}-\epsilon^{2}-t_{2}^{2}, \\
& r_{2} r_{3} \cos \left(\theta_{2}-\theta_{3}\right)=L^{2}-\epsilon^{2}-t_{1}^{2}, \\
& r_{2} r_{4} \cos \left(\theta_{2}-\theta_{4}\right)=l L-\epsilon^{2}-t_{1} t_{2}-i \epsilon\left(-t_{1}+t_{2}\right), \\
& r_{3} r_{4} \cos \left(\theta_{3}-\theta_{4}\right)=l L+\epsilon^{2}-t_{1} t_{2}+i \epsilon\left(t_{1}+t_{2}\right) .
\end{aligned}
$$

After performing this analytic continuation, we take the limit $\epsilon \rightarrow 0$. In this limit, the 4 point function on $\Sigma_{1}$ is given by

$$
\left\langle\phi\left(r_{1}, \theta_{1}\right) \phi\left(r_{2}, \theta_{2}\right) \phi\left(r_{3}, \theta_{3}\right) \phi\left(r_{4}, \theta_{4}\right)\right\rangle_{\Sigma_{1}}=\frac{1}{256 \pi^{4} \epsilon^{4}}+\mathcal{O}\left(\epsilon^{0}\right)
$$

On the other hand, the leading term of 8 point function of $\phi$ on $\Sigma_{2}$ is $\mathcal{O}\left(\epsilon^{-8}\right)$ in this limit. In the region $t_{2} \leq l$, the leading term of 8-point function of $\phi$ is given by

$$
\left\langle\phi^{8}\right\rangle_{\Sigma_{2}} \sim \frac{L^{2}+t_{1}^{2}}{131072 \pi^{8} t_{1}^{2} \epsilon^{8}},
$$

where $\left\langle\phi^{8}\right\rangle_{\Sigma_{2}}$ denotes the 8 point function on $\Sigma_{2}$. In the region $t_{2} \leq l$, their leading terms are given by

$$
\left\langle\phi^{8}\right\rangle_{\Sigma_{2}} \sim \frac{\left(l^{2}+t_{2}^{2}\right)\left(L^{2}+t_{1}^{2}\right)}{262144 \pi^{8} t_{2}^{2} t_{1}^{2} \epsilon^{8}}
$$

In this limit, only spesific propagators can contribute to it. Their leading term is $\mathcal{O}\left(\epsilon^{-2}\right) . \quad\left\langle\phi\left(r_{1}, \theta_{1}\right) \phi\left(r_{4}, \theta_{4}\right)\right\rangle_{\Sigma_{2}},\left\langle\phi\left(r_{1}, \theta_{1}+2 \pi\right) \phi\left(r_{4}, \theta_{4}+2 \pi\right)\right\rangle_{\Sigma_{2}},\left\langle\phi\left(r_{2}, \theta_{2}\right) \phi\left(r_{3}, \theta_{3}\right)\right\rangle_{\Sigma_{2}}$, $\left\langle\phi\left(r_{2}, \theta_{2}\right) \phi\left(r_{3}, \theta_{3}+2 \pi\right)\right\rangle_{\Sigma_{2}},\left\langle\phi\left(r_{2}, \theta_{2}+2 \pi\right) \phi\left(r_{3}, \theta_{3}\right)\right\rangle_{\Sigma_{2}}$ and $\left\langle\phi\left(r_{2}, \theta_{2}+2 \pi\right) \phi\left(r_{3}, \theta_{3}+2 \pi\right)\right\rangle_{\Sigma_{2}}$ 
can contribute to the leading term of 8 point function in the region $t_{2} \leq l$. In this limit, they are given by

$$
\begin{aligned}
& \left\langle\phi\left(r_{2}, \theta_{2}\right) \phi\left(r_{3}, \theta_{3}\right)\right\rangle_{\Sigma_{2}}=\left\langle\phi\left(r_{2}, \theta_{2}+2 \pi\right) \phi\left(r_{3}, \theta_{3}+2 \pi\right)\right\rangle_{\Sigma_{2}}=\frac{L+t_{1}}{32 \pi^{2} t_{1} \epsilon^{2}}+\mathcal{O}\left(\epsilon^{-1}\right), \\
& \left\langle\phi\left(r_{2}, \theta_{2}\right) \phi\left(r_{3}, \theta_{3}+2 \pi\right)\right\rangle_{\Sigma_{2}}=\left\langle\phi\left(r_{2}, \theta_{2}+2 \pi\right) \phi\left(r_{3}, \theta_{3}\right)\right\rangle_{\Sigma_{2}}=\frac{-L+t_{1}}{32 \pi^{2} t_{1} \epsilon^{2}}+\mathcal{O}\left(\epsilon^{-1}\right), \\
& \left\langle\phi\left(r_{1}, \theta_{1}\right) \phi\left(r_{4}, \theta_{4}\right)\right\rangle_{\Sigma_{2}}=\left\langle\phi\left(r_{1}, \theta_{1}+2 \pi\right) \phi\left(r_{4}, \theta_{4}+2 \pi\right)\right\rangle_{\Sigma_{2}}=\frac{1}{16 \pi^{2} \epsilon^{2}}+\mathcal{O}\left(\epsilon^{0}\right) .
\end{aligned}
$$

In the region $t_{2} \geq l$, the contribution from $\left\langle\phi\left(r_{2}, \theta_{2}\right) \phi\left(r_{3}, \theta_{3}\right)\right\rangle_{\Sigma_{2}}$, $\left\langle\phi\left(r_{2}, \theta_{2}\right) \phi\left(r_{3}, \theta_{3}+2 \pi\right)\right\rangle_{\Sigma_{2}},\left\langle\phi\left(r_{2}, \theta_{2}+2 \pi\right) \phi\left(r_{3}, \theta_{3}\right)\right\rangle_{\Sigma_{2}}$ and $\left\langle\phi\left(r_{2}, \theta_{2}+2 \pi\right) \phi\left(r_{3}, \theta_{3}+2 \pi\right)\right\rangle_{\Sigma_{2}}$ does not change. On the other hand, the contribution from $\left\langle\phi\left(r_{1}, \theta_{1}\right) \phi\left(r_{4}, \theta_{4}\right)\right\rangle_{\Sigma_{2}}$, $\left\langle\phi\left(r_{1}, \theta_{1}+2 \pi\right) \phi\left(r_{4}, \theta_{4}+2 \pi\right)\right\rangle_{\Sigma_{2}}$ changes as follows,

$$
\left\langle\phi\left(r_{1}, \theta_{1}\right) \phi\left(r_{4}, \theta_{4}\right)\right\rangle_{\Sigma_{2}}=\left\langle\phi\left(r_{1}, \theta_{1}+2 \pi\right) \phi\left(r_{4}, \theta_{4}+2 \pi\right)\right\rangle_{\Sigma_{2}}=\frac{l+t_{2}}{32 \pi^{2} t_{2} \epsilon^{2}}+\mathcal{O}\left(\epsilon^{-1}\right),
$$

$\left\langle\phi\left(r_{1}, \theta_{1}+2 \pi\right) \phi\left(r_{4}, \theta_{4}\right)\right\rangle_{\Sigma_{2}}$ and $\left\langle\phi\left(r_{1}, \theta_{1}+2 \pi\right) \phi\left(r_{4}, \theta_{4}+2 \pi\right)\right\rangle_{\Sigma_{2}}$ can also contribute to the leading term of the 8-point function of $\phi$. They are given

$$
\left\langle\phi\left(r_{1}, \theta_{1}\right) \phi\left(r_{4}, \theta_{4}+2 \pi\right)\right\rangle_{\Sigma_{2}}=\left\langle\phi\left(r_{1}, \theta_{1}\right) \phi\left(r_{4}, \theta_{4}+2 \pi\right)\right\rangle_{\Sigma_{2}}=\frac{-l+t_{2}}{32 \pi^{2} t_{2} \epsilon^{2}}+\mathcal{O}\left(\epsilon^{-1}\right) .
$$

The contribution from the other propagators is at most $\mathcal{O}\left(\epsilon^{-1}\right)$. Then they can not contribute to it. In the limit $\epsilon \rightarrow 0, \Delta S_{A}^{(2)}$ for this state is given by

$$
\Delta S_{A}^{(2)}= \begin{cases}\log \left[\frac{2 t_{1}^{2}}{L^{2}+t_{1}^{2}}\right] & t_{2} \leq l \\ \log \left[\frac{4 t_{1}^{2} t_{2}^{2}}{\left(l^{2}+t_{2}^{2}\right)\left(L^{2}+t_{1}^{2}\right)}\right] & t_{2} \geq l\end{cases}
$$

Its time evolution is plotted in figure 9 . The final value of $\Delta S_{A}^{(2)}$ is given by

$$
\Delta S_{A}^{(2)}=\log 2+\log \left[\frac{2 t_{1}^{2}}{L^{2}+t_{1}^{2}}\right]
$$

We are able to control its final value by turning parameters $t_{1}$ and $L$. Its maximum value is given by

$$
\Delta S_{A}^{(2) \max }=2 \log 2 .
$$

\subsubsection{Simultaneous case}

Let us consider the case where we act two operators $\phi \phi$ simultaneously on the ground state. They are located at $x^{1}=-l$ and $x^{1}=-L$ respectively. Here, we assume that $L>l$. We investigate the evolution of second Rényi entropy with $t_{1}$ for this state. ${ }^{7}$ We can compute it as we explained above. We just have to change $t_{2}$ to $t_{1}$ in (3.21). Therefore we skip the detail of the calculation. Of course, we perform the analytic continuation similar to that in (3.21) and take the limit $\epsilon \rightarrow 0$.

\footnotetext{
${ }^{7}$ If we act one of them on the ground state at $t=-t_{1}$ and another one on it at $t=-t_{1}+c(c \geq 0)$, we can investigate the evolution of $\Delta S_{A}^{(2)}$. Here, we think about the simplest case.
} 


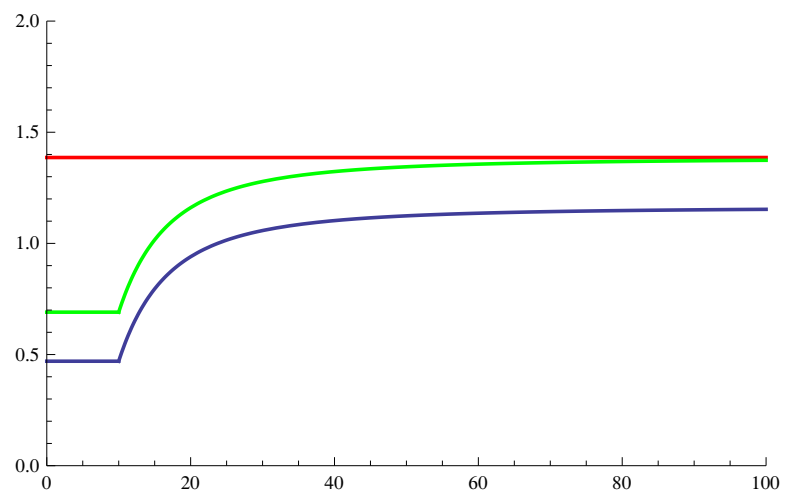

Figure 9. Plots of the evolution of $\Delta S_{A}^{(2)}$ with $t_{2}$ in the limit $\epsilon \rightarrow 0$. The vertical axis corresponds to $\Delta S_{A}^{(2)}$. The horizontal axis corresponds to $t_{2}$. Here we chose $l=10$. The blue curve corresponds to the time evolution of $\Delta S_{A}^{(2)}$ for $\phi \phi|0\rangle$, where one of two scalar fields is located at $t=-t_{1}=-10$. The other is located at $x^{1}=-L=-5$. The green curve corresponds to the time evolution of $\Delta S_{A}^{(2)}$ for $\phi \phi|0\rangle$ where one of two scalar fields is located at $t=-t_{1}=-100$. The other is located at $x^{1}=-L=-5$. The red curve corresponds to $2 \log 2$.

The time evolution of its second Rényi entanglement entropy is given by

$$
\Delta S_{A}^{(2)}= \begin{cases}0 & 0 \leq t_{1} \leq l \\ \log \left[\frac{2 t_{1}^{2}}{l^{2}+t_{1}^{2}}\right] & t_{1} \geq l, \\ \log \left[\frac{2 t_{1}^{2}}{l^{2}+t_{1}^{2}}\right]+\log \left[\frac{2 t_{1}^{2}}{L^{2}+t_{1}^{2}}\right] & t_{1} \geq L .\end{cases}
$$

The evolution of $\Delta S_{A}^{(2)}$ with $t_{1}$ is plotted in figure 10. If the entropies for the states which are excited by $\phi\left(-t_{1},-L\right)$ and $\phi\left(-t_{1},-l\right)$ are defined by $\Delta S_{A}^{(2), 1}$ and $\Delta S_{A}^{(2), 2}$ respectively, then the entropy for the state generated by acting both of them on the ground state is given by the sum of those entropies,

$$
\Delta S_{A}^{(2)}=\Delta S_{A}^{(2), 1}+\Delta S_{A}^{(2), 2} .
$$

If we take the limit $t_{1} \rightarrow \infty$, the final value of $\Delta S_{A}^{(2)}$ is given by

$$
\Delta S_{A}^{(2) f}=2 \log 2 .
$$

\section{Entangled pair interpretation}

In the previous section, we investigate the time evolution of $\Delta S_{A}^{(n)}$. Here we will interpret the time evolution of $\Delta S_{A}^{(n)}$ in terms of the relativistic propagation of entangled quanta. Moreover we will acquire $\Delta S_{A}^{(n) f}$ for the state defined by acting the local operator : $\phi^{k}$ : on the ground state under this interpretation.

We will also explain our new results. One of them is the large $k$ behavior of $\Delta S_{A}^{(n) f}$ for the state defined by acting the local operator $: \phi^{k}$ : on the ground state. We will find the sum rule for (Rényi) entanglement entropies for the state defined by acting several operators on the ground state. We assume that they are given by : $\phi^{k_{i}}$ : and located separately. We will argue that $\Delta S_{A}^{(n) f}$ for the state defined by acting specific operators on the ground state is given by (4.11). We will also argue that the sum rule can be generalized. 


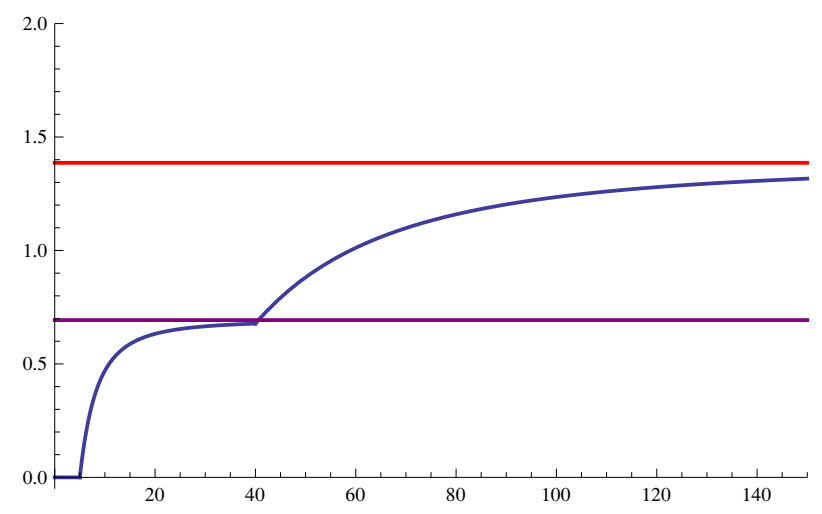

Figure 10. Plots of the evolution of $\Delta S_{A}^{(2)}$ with $t_{1}$ in the limit $\epsilon \rightarrow 0$. The vertical axis corresponds to $\Delta S_{A}^{(2)}$. The horizontal axis corresponds to $t_{1}$. Here we chose $l=5$ and $L=40$. The blue curve is the time evolution of $\Delta S_{A}^{(2)}$ for $\phi \phi|0\rangle$ where one of the two scalar fields is spatially located at $x^{1}=-l=-5$. Another one is located at $x^{1}=-L=-40$ The purple curve is $\log 2$. The red curve is $2 \log 2$.
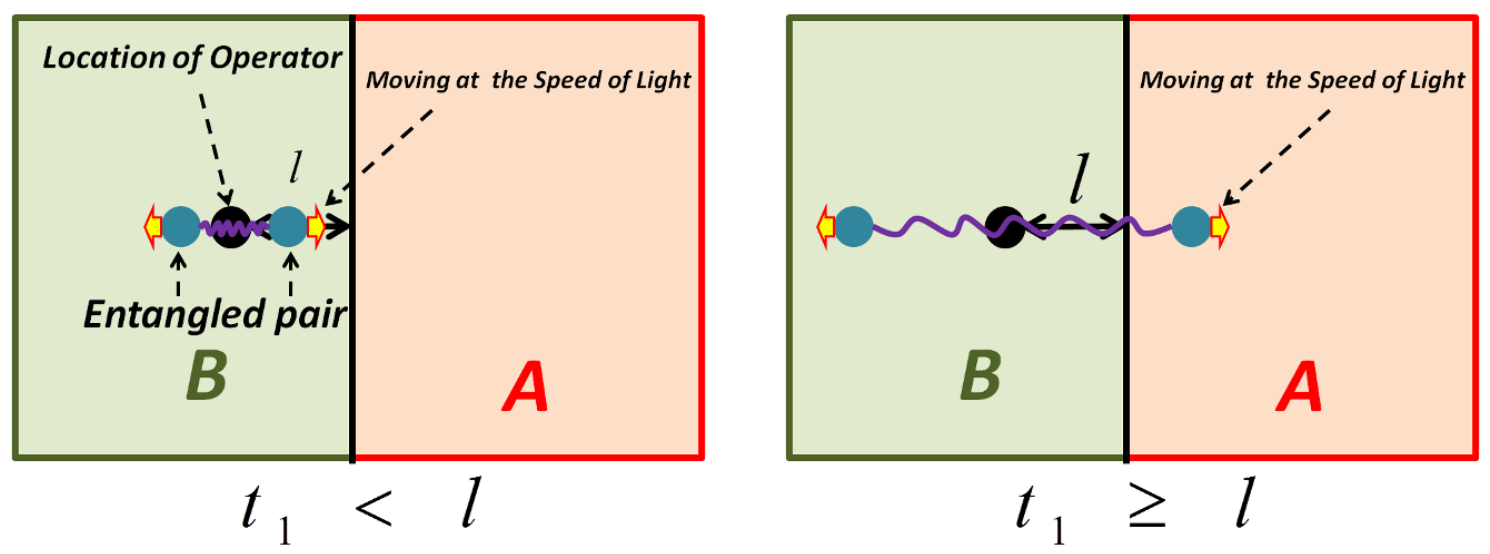

Figure 11. The schematic explanation for the time evolution of $\Delta S_{A}^{(n)}$. If $t_{1}<l$, each of an entangled pair is included in the region B. If $t_{1} \geq l$, two quanta are included in A and B respectively.

\subsection{A physical interpretation of $\Delta S_{A}^{(n)}$ in two dimensions}

We investigated the time evolution of $\Delta S_{A}^{(n)}$ for the locally excited states in 2,4 and 6 dimensional spacetime in the previous section. Here we interpret their time evolutions in terms of the relativistic propagation of quasi-particles.

Let us consider the result of $\mathcal{N}^{-1}\left(e^{i \alpha \phi}+e^{-i \alpha \phi}\right)|0\rangle$ in two dimensions. The time evolution of $\Delta S_{A}^{(2)}$ for this state corresponds to the blue curve in figure 5. In this figure, the amount of $\Delta S_{A}^{(2)}$ can not increase until $t_{1}=l$. It drastically increases at $t_{1}=l$ and approaches the constant value $\log 2$ at late time.

We can interpret its time evolution in terms of the relativistic propagation of entangled quanta as follows. First, a pair of two quanta appears at the point where $\left(e^{i \alpha \phi}+e^{-i \alpha \phi}\right)$ is located as in figure 11. Here we assume it is inserted into the complement B of the subsystem A. Those two quanta are entangled with each other and we call this pair an 
entangled pair. Each of two quanta propagates in the opposite direction at the speed of light. Until $t_{1}=l$, two quanta remain to stay in the region $\mathrm{B}$. Then the quantum entanglement between them is not able to contribute to $\Delta S_{A}^{(2)}$. When one of them reaches at the boundary $\partial A$ of the subsystem $\mathrm{A}$, the quantum entanglement between them begins to contribute to second Rényi entanglement entropy between A and B. Since they propagate at the speed of light, one of them can reaches at $\partial A$ at $t_{1}=l$. After it reaches at $\partial A$, the entanglement between them can contribute to $\Delta S_{A}^{(2)}$. We chose a half of total space as the subsystem A. Therefore, once one of them enters the subsystem A, it can not go out there. Since each of entangled pair remains to stay in A and B respectively, $\Delta S_{A}^{(2)}$ can approach a finite constant. In particular, these quanta can propagate in only one direction respectively in two dimensional examples. Therefore $\Delta S_{A}^{(2)}$ suddenly approaches a nontrivial constant at $t_{1}=l$. In higher dimensional examples $(d>1)$, those quanta can propagate in generic direction. Then $\Delta S_{A}^{(n)}$ approach some constant values mildly as in figure 7 .

Moreover we are able to find out why $\Delta S_{A}^{(2) f}$ is given by $\log 2$ under this interpretation. We decompose $\phi$ into $\phi_{L}$ and $\phi_{R}$,

$$
\phi=\phi_{L}+\phi_{R}
$$

where $\phi_{L}$ and $\phi_{R}$ respectively correspond to modes which are moving towards the left direction $\left(x^{1}<0\right)$ and the right direction $\left(x^{1}>0\right)$. Therefore this locally excited state is given by

$$
|\Psi\rangle=\frac{1}{\sqrt{2}}\left|e^{-i \alpha \phi_{R}}\right\rangle_{R} \otimes\left|e^{-i \alpha \phi_{L}}\right\rangle_{L}+\frac{1}{\sqrt{2}}\left|e^{i \alpha \phi_{R}}\right\rangle_{R} \otimes\left|e^{i \alpha \phi_{L}}\right\rangle_{L},
$$

where $|\Psi\rangle$ is normalized so as to $\langle\Psi \mid \Psi\rangle=1$. This locally excited state is an EPR state (a maximally entangled state). Therefore $\Delta S_{A}^{(2) f}$ is given by $\log 2$.

For any $n$, we can obtain its (Rényi) entanglement entropies. Its reduced matrix is given by

$$
\rho_{A}=\frac{1}{2}\left(\begin{array}{ll}
1 & 0 \\
0 & 1
\end{array}\right),
$$

and for any $n, \rho_{A}^{n}$ is given by

$$
\rho_{A}^{n}=\frac{1}{2^{n}}\left(\begin{array}{ll}
1 & 0 \\
0 & 1
\end{array}\right) .
$$

The definition of (Rényi) entanglement entropies are given in (2.7). Those for this EPR state do not depend $n$. Therefore they are given by

$$
\Delta S_{A}^{(n)}=\log 2 .
$$

Let us apply this interpretation for other results in two dimensional cases. In the case where excited states are defined by acting the derivatives of $\phi$ mentioned above and $: e^{ \pm i \alpha \phi}:|0\rangle$ on the ground state, they are given by products states. Therefore $\Delta S_{A}^{(n)}$ vanishes. In the next subsection, we apply the entangled pair interpretation for the results in higher dimensional cases.

\subsection{A physical interpretation in higher dimensions $(d>1)$}

Let us apply the entangled pair representation for results in higher dimensional cases. We will obtain $\Delta S_{A}^{(n) f}$ for states defined by acting : $\phi^{k}$ : on the ground state. 
We decompose $\phi$ into $\phi_{L}$ and $\phi_{R}$ as in (4.1). Using this decomposition, the excited state defined by acting : $\phi^{k}$ : on the ground state is given by

$$
|\Psi\rangle=\mathcal{N}^{-1}: \phi^{k}:|0\rangle=\mathcal{N}^{-1} \sum_{m=0}^{k}{ }_{k} C_{m} \phi_{R}^{m}|0\rangle_{R} \otimes \phi_{L}^{k-m}|0\rangle_{L},
$$

where $\mathcal{N}$ is the normalization factor and $|0\rangle_{R}$ and $|0\rangle_{L}$ are the vacuum for the right moving mode and the left moving mode respectively. Moreover we define new bases by,

$$
\begin{aligned}
|j\rangle_{R} & =\frac{1}{\sqrt{j !}}\left(\phi_{R}\right)^{j}|0\rangle_{R}, \\
|j\rangle_{L} & =\frac{1}{\sqrt{j !}}\left(\phi_{L}\right)^{j}|0\rangle_{L},
\end{aligned}
$$

where they are normalized so as to $\langle i \mid j\rangle_{L, R}=\delta_{i j}$. And $|\Psi\rangle$ is also normalized so as to $\langle\Psi \mid \Psi\rangle=1$. After normalized it, it is given by

$$
|\Psi\rangle=\frac{1}{2^{\frac{k}{2}}} \sum_{m=0}^{k} \sqrt{{ }_{k} C_{m}}|m\rangle_{A} \otimes|k-m\rangle_{B} .
$$

If we take the limit $t_{1} \rightarrow \infty$, we can identify the degrees of freedom of right moving modes and left moving modes with those in the subsystem A and B respectively. Therefore tracing out the degrees of freedom in the subsysten $\mathrm{B}$ is equivalent to tracing out those of left moving modes. After tracing out the degrees of freedom in the subsystem B, its reduced density matrix is give by

$$
\rho_{A, k}=\frac{1}{2^{k}}\left(\begin{array}{ccccc}
{ }_{k} C_{0} & 0 & 0 & \cdots & 0 \\
0 & { }_{k} C_{1} & 0 & \cdots & 0 \\
\vdots & \vdots & \ddots & \vdots & \vdots \\
0 & 0 & 0 & \cdots & { }_{k} C_{k}
\end{array}\right)
$$

where ${ }_{k} C_{m}=\frac{k !}{(k-m) ! m !}$. It is given by the binominal distribution.

By using the matrix in (4.9), we can obtain its explicit form for each $\mathrm{k}$ as follows,

$$
\begin{aligned}
\rho_{A, 1}=\frac{1}{2}\left(\begin{array}{ll}
1 & 0 \\
0 & 1
\end{array}\right), & \rho_{A, 2}=\frac{1}{4}\left(\begin{array}{lll}
1 & 0 & 0 \\
0 & 2 & 0 \\
0 & 0 & 1
\end{array}\right), \\
\rho_{A, 3}=\frac{1}{8}\left(\begin{array}{llll}
1 & 0 & 0 & 0 \\
0 & 3 & 0 & 0 \\
0 & 0 & 3 & 0 \\
0 & 0 & 0 & 1
\end{array}\right), & \rho_{A, 4}=\frac{1}{16}\left(\begin{array}{llllll}
1 & 0 & 0 & 0 & 0 \\
0 & 4 & 0 & 0 & 0 \\
0 & 0 & 6 & 0 & 0 \\
0 & 0 & 0 & 4 & 0 \\
0 & 0 & 0 & 0 & 1
\end{array}\right), \\
\rho_{A, 5}=\frac{1}{32}\left(\begin{array}{cccccc}
1 & 0 & 0 & 0 & 0 & 0 \\
0 & 5 & 0 & 0 & 0 & 0 \\
0 & 0 & 10 & 0 & 0 & 0 \\
0 & 0 & 0 & 10 & 0 & 0 \\
0 & 0 & 0 & 0 & 5 & 0 \\
0 & 0 & 0 & 0 & 0 & 1
\end{array}\right) . &
\end{aligned}
$$




\begin{tabular}{|c|c|c|c|c|c|c|}
\hline Entropy & $l$ & $m=1$ & $m=2$ & $m=3$ & $\cdots$ & $m=j$ \\
\hline \multirow{4}{*}{$\Delta S_{A, m}^{(l) f}$} & 2 & $\log 2$ & $\log \frac{8}{3}$ & $\log \frac{16}{5}$ & $\cdots$ & $-\log \left(\frac{1}{2^{2 j}} \sum_{i=0}^{j}\left({ }_{j} C_{i}\right)^{2}\right)$ \\
\cline { 2 - 7 } & 3 & $\log 2$ & $\frac{1}{2} \log \frac{32}{5}$ & $\frac{1}{2} \log \frac{64}{7}$ & $\cdots$ & $\frac{-1}{2} \log \left(\frac{1}{2^{3 j}} \sum_{i=0}^{j}\left({ }_{j} C_{i}\right)^{3}\right)$ \\
\cline { 2 - 7 } & $\vdots$ & $\vdots$ & $\vdots$ & $\vdots$ & $\vdots$ & $\vdots$ \\
\cline { 2 - 7 } & $n$ & $\log 2$ & $\frac{1}{n-1} \log \frac{2^{2 n-1}}{2^{n-1}+1}$ & $\frac{1}{n-1} \log \frac{2^{3 n-1}}{3^{n}+1}$ & $\cdots$ & $\frac{1}{1-n} \log \left(\frac{1}{2^{n j}} \sum_{i=0}^{j}\left({ }_{j} C_{i}\right)^{n}\right)$ \\
\hline \multirow{2}{*}{$\Delta S_{A, j}$} & 1 & $\log 2$ & $\frac{3}{2} \log 2$ & $\frac{3}{4} \log \frac{16}{3}$ & $\cdots$ & $j \cdot \log 2-\frac{1}{2^{j}} \sum_{i=0}^{j}{ }_{j} C_{i} \log { }_{j} C_{i}$ \\
\hline
\end{tabular}

Table 1. The value of $\Delta S_{A, m}^{(l) f}$ for the free massless scalar field theory in dimensions higher than two. Here $l$ is the number of sheet. $m$ is the number of $\phi$.

Substituting $\rho_{A, j}$ in (4.9) into (2.7), we are able to acquire $n$-th Rényi entanglement entropy for the locally excited state defined by acting $: \phi^{k}:$ on the ground state,

$$
\Delta S_{A, k}^{(n) f}=\frac{1}{1-n} \log \left(\frac{1}{2^{n k}} \sum_{m}^{k}\left({ }_{k} C_{m}\right)^{n}\right) .
$$

Its reduced density matrix is given by the binomial distribution.

We can derive the explicit results for each $k, n$ from the formula in (4.11). They do not depend on the spacetime dimension as long as it is higher than 2. As you see later, they are consistent with results which we will obtain by the replica method. These results are summarized in table 1 . In any spacetime dimensions, the final values of (Rényi) entanglement entropies for the state which is excited by $: \phi^{k}$ : are given by (4.11).

In $n=2$ case, the formula in (4.11) is simplified,

$$
\Delta S_{A, k}^{(2) f}=\sum_{m=1}^{k} \log \frac{2 m}{2 m-1}
$$

$\Delta S_{A, 1}^{(n)}$ does not depend on the number of sheets. It is equivalent to (Rényi) entanglement entropy for an EPR states (maximally excited state). Using the formula in (4.11), we can compute $\Delta S_{A, k}^{(n) f}$ for the state defined by acting : $\phi^{k}$ : on the ground state. As we will see later, the formula in (4.11) gives a characterization of operators.

We define (Rényi) entanglement entropies of operators by the final value of (Rényi) entanglement entropies for the state defined by acting them on the ground state. They characterize operators from the view point of the quantum entanglement and they are independent of the conformal dimension of operators. ${ }^{8}$ (Rényi) entanglement entropies operators are related to the number of entangled pairs which those operators are able to create at least in the free massless scalar field theory.

\footnotetext{
${ }^{8}$ In two dimensional cases, we have seen that $\Delta S_{A}^{(n) f}$ do not depend on conformal dimensions of operators. The conformal dimension of $\mathcal{O}_{1}=: e^{i \alpha \phi}:$ is the same as that of $\mathcal{O}_{2}=: e^{i \alpha \phi}:+: e^{-i \alpha \phi}:$. However $\Delta S_{A}^{(n) f}$ for $\mathcal{O}_{1}$ is not the same as that for $\mathcal{O}_{2}$.
} 


\subsubsection{Large $k$ behavior}

Here we explain one of the new results. We would like to comment on the large $k$ behavior of $\Delta S_{A}^{(n) f}$. (Rényi) entanglement entropies of : $\phi^{k}$ : are given by (4.11). Therefore in the large $k$ limit we can approximate its reduced density matrix by the normal distribution as follows

$$
\rho_{A}=\frac{{ }_{k} C_{x}}{2^{k}} \sim \sqrt{\frac{a}{k \pi}} e^{-\frac{a\left(x-\frac{k}{2}\right)^{2}}{k}},
$$

where $a$ is a certain positive constant. By using this approximation, we would like to evaluate $\Delta S_{A, j}^{(n) f}$ in table 1. $\Delta S_{A, j}^{(n)}$ are given by

$$
\Delta S_{A}^{(n) f} \sim \frac{1}{2} \log k .
$$

They do not depend on the number of sheets.

\subsubsection{Generalization of $\Delta S_{A, j}^{(n) f}$}

We explained (Rényi) entanglement entropies of : $\phi^{k}:$ up to here. Let us consider (Rényi) entanglement entropies for the states defined by acting specific operators on the ground state. We consider specific operators such as : $\left(\partial^{l} \phi\right)^{k}$ :. They are composed of single-species operators such as $\phi, \partial \phi, \partial \partial \phi$ and so on. We call them S-operators. In the argument above, we assumed that operators can be decomposed to left moving modes and right moving modes at late time. Under this assumption, we did not use the detailed feature of local operator $\phi$. Therefore, we argue that we are able to decompose these operators $(\phi, \partial \phi$, $\partial \partial \phi, \cdots)$ into the right moving mode and the left moving mode when states are excited by S-operators. Therefore in the free massless scalar field theory in any dimensions, (Rényi) entanglement entropies of S-operators are given by the formula in (4.11).

In summary, we argue that (Rényi) entanglement entropies of S-operators should be given by (4.11). This implies that they are independent of conformal dimensions of operators. (Rényi) entanglement entropies of operators characterize operators from the viewpoint of quantum entanglement. ${ }^{9}$ We have explicitly computed (Rényi) entanglement entropies of $(\partial \phi)^{j}$, for $n=2,3$ in 4 dimensions. We found that they are consistent with the results we obtained under this assumption. Moreover, $\Delta S_{A}^{(n) f}$ which we obtain under the entangled pair interpretation agree with the result which we will obtain by the replica method.

\subsection{Sum rule of (Rényi) entanglement entropies of operators}

We will interpret the final values of (Rényi) entanglement entropies for states by acting various operators on the ground state in terms of entangled pairs. First, we think about $\Delta S_{A}^{(n)}$ for $\mathcal{N}^{-1} \mathcal{T}: \phi_{1}^{m_{1}}:: \phi_{2}^{m_{2}}:|0\rangle$. Those operators are located separately. We assume $: \phi_{1}^{m_{1}}:,: \phi_{2}^{m_{2}}$ : can be independently decomposed into left moving modes and right moving modes as follows,

$$
\begin{aligned}
& \phi_{1}=\phi_{L, 1}+\phi_{R, 1}, \\
& \phi_{2}=\phi_{L, 2}+\phi_{R, 2},
\end{aligned}
$$

\footnotetext{
${ }^{9}$ The authors in $[72]$ found the entanglement of operator are related to the quantum dimension in rational CFTs on the 2 dimensional space
} 
where $\phi_{L, 1}$ and $\phi_{R, 1}$ are independent of other operators $\phi_{L, 2}, \phi_{R, 2}$. The annihilation and creation operators of $\phi_{L, R, 1}$ can commute with those of $\phi_{L, R, 2} \cdot{ }^{10}$

Under this assumption, the normalized excited state is given by

$$
\begin{aligned}
|\Psi\rangle & =\mathcal{N}^{-1} \mathcal{T}: \phi_{1}^{m_{1}}:: \phi_{2}^{m_{2}}:|0\rangle \\
& =\frac{1}{2^{\frac{m_{1}}{2}} 2^{\frac{m_{2}}{2}}} \sum_{k_{1}=0}^{m_{1}} \sum_{k_{1}=0}^{m_{2}} \sqrt{m_{1} C_{k_{1}} m_{2} C_{k_{2}}}\left|m_{1}-k_{1}, m_{2}-k_{2}\right\rangle_{L} \otimes\left|k_{1}, k_{2}\right\rangle_{R}
\end{aligned}
$$

where $\mathcal{T}$ is a time ordering operator.

In any dimensions, the final values of its (Rényi) entanglement entropy are given by

$$
\Delta S_{A}^{(n) f}=\Delta S_{A, m_{1}}^{(n) f}+\Delta S_{A, m_{2}}^{(n) f}
$$

where $\Delta S_{A, m_{1}}^{(n) f}$ and $\Delta S_{A, m_{2}}^{(n) f}$ are given by (4.11). They are (Rényi) entanglement entropies of : $\phi^{m_{1}}$ : and : $\phi^{m_{2}}$ : respectively. $\Delta S_{A}^{(n) f}$ are given by the sum of $\Delta S_{A, m_{1}}^{(n) f}$ and $\Delta S_{A, m_{2}}^{(n) f}$. When $m_{1}, m_{2}$ are 1 respectively in $n=2$ case, $\Delta S_{A}^{(2) f}$ is consistent with the result which we obtained in the previous section.

The sum rule in (4.17) can be generalized. We prepare the locally excited state defined by acting various operators $\mathcal{O}^{i}$ on the ground state. Those operators $\mathcal{O}^{i}(i=1 \sim q)$ are given by : $\phi^{k_{i}}:$. We assume they are located separately. In any dimensions $(d>1), \Delta S_{A}^{(n) f}$ at late time for the state generated by acting them on the ground state are given by

$$
\Delta S_{A}^{(n) f}=\sum_{i=1}^{q} \Delta S_{A, k_{i}}^{(n) f i},
$$

where $\Delta S_{A}^{(n) f i}$ are given by (Rényi) entanglement entropies of the local operator $\mathcal{O}^{i}$. As you see later, the result in (4.18) agrees with the result which we obtain by using the replica method.

\subsubsection{Generalized sum rule}

We would like to generalize the sum rule of (Rényi) entanglement entropies of operators to that in the more general case where operators are not limited to $: \phi^{k_{i}}:$. We did not use the distinctive feature of $\phi$ when we found the sum rule of $\Delta S_{A}^{(n) f}$. We assumed that $\phi$ can be decomposed into the left moving mode and the right moving mode. If the location of $\phi_{1}$ is separated from the location of $\phi_{2}, \phi_{1}$ is independent of $\phi_{2}$. It is expected that we can apply this assumption for (Rényi) entanglement entropies of operators other than : $\phi^{k}$ :. Therefore we argue that if the state is defined by acting various general operators $\mathcal{O}^{i}$ on the ground state, $\Delta S_{A}^{(n) f}$ for it should obey the sum rule which is given by (4.18). Here, we assume that $\mathcal{O}^{i}$ are located separately. In other words, $\Delta S_{A}^{(n) f}$ for that state are given by the sum of (Rényi) entanglement entropies of those operators in any dimensions.

\footnotetext{
${ }^{10}$ If these two operators are located on the light cone, it is not clear that they are independent of each other. However in the replica method, it does not seem that $\Delta S_{A}^{(n)}$ shows nontrivial behavior even if they are located on the light cone.
} 
In summary, an excited state is given by

$$
|\Psi\rangle=\prod_{i=1}^{q} \mathcal{O}^{i}\left(t_{i}, x_{i}\right)|0\rangle,
$$

where the locations of operators are separated from the locations of operators other than itself. We assume that $\mathcal{O}^{i}$ are general operators.

In the $d+1(\geq 2)$ dimensional massless free scalar field theory, its (Rényi) entanglement entropies are given by

$$
\Delta S_{A}^{(n) f}=\sum_{i=1}^{q} \Delta S_{A}^{(n) f i},
$$

where $\Delta S^{(n) f i}$ are (Rényi) entanglement entropies of $\mathcal{O}^{i}\left(t_{i}, x_{i}\right)$.

\section{General argument by using propagators}

In this section we would like to explain our new results. First we find the explanation which shows that (Rényi) entanglement entropies of: $\phi^{k}$ : are given by entropies whose reduced density matrices are given by the binomial distribution by the replica method. We will also obtain the sum rule which (Rényi) entanglement entropies for excited states defined by acting various operators on the ground state. We assume that they are located separately. Moreover we argue that (Rényi) entanglement entropies of the specific operators $\left(\partial^{m} \phi\right)^{n}$ are given by (Rényi) entanglement entropies whose reduced density matrices are given by the binomial distribution. We will also argue that $\Delta S_{A}^{(n)}$ for excited states defined by acting various operators on the ground state obey the same sum rule. Those results which we obtain by the replica trick will agree with the results which we obtained in terms of entangled pairs. Finally we will comment on the effects of a conical singularity on the boundary of the subsystem A. On $\Sigma_{n}$ there is a conical singularity on the boundary of the subsystem A. At first, it seems that the conical singularity can affect (Rényi) entanglement entropies for locally excited states. However it can not affect them as we will explain later.

\subsection{Properties of propagators}

In the free massless scalar field theory, we have to calculate propagators to compute the correlation functions of local operators. After computing $\Delta S_{A}^{(n)}$ by the replica method, we perform the analytic continuation in (2.5) to real time. By taking the limit $\epsilon \rightarrow 0$, the calculations of $\Delta S_{A}^{(n)}$ are simplified due to a distinctive feature of propagators. We assume local operators are located at $\left(r_{1}, \theta_{1}\right)$ and $\left(r_{2}, \theta_{2}\right)$ on $\Sigma_{1}$ as in figure 1 .

Only specific propagators on $\Sigma_{n}$ can dominantly contribute to the $2 n$ point correlation function of local operators after performing the analytic continuation in (2.5). We define these dominant propagators on $\Sigma_{n}$ by $D^{(n)}$. As in figure 12, the propagators which are aligned on a circle are dominant propagators. This figure shows the configuration of dominant propagators on $\Sigma_{4}$. This circle is made of $2 n$ propagators. Those $2 n$ two point functions necessarily include contribution from the propagators between two points on the same 

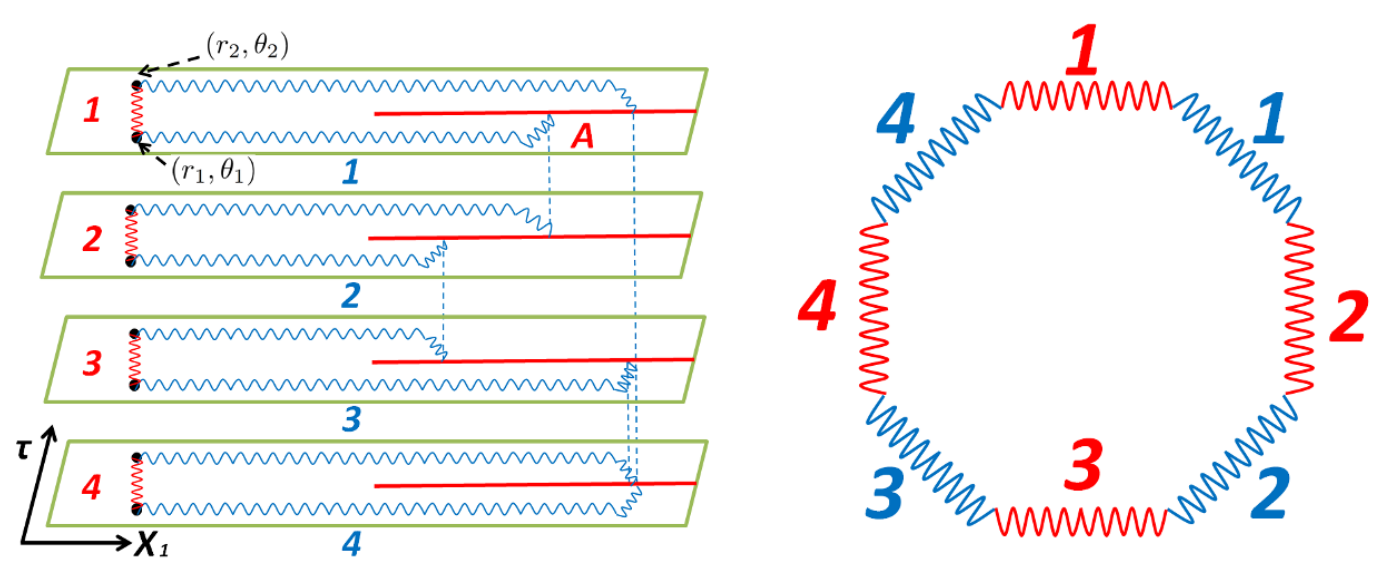

Figure 12. The circle of dominant propagators on a 4 sheeted geometry. There are 4 blue wavy lines and red wavy lines respectively. Dots on $\Sigma_{4}$ are points where operators are located.

sheet. They respectively correspond to the 4 red wavy lines in figure 12 . The other dominant propagators are those between $\left(r_{1}, \theta_{1, k}\right)$ and $\left(r_{2}, \theta_{2, k+1}\right) \quad\left(k=1, \cdots, n, \theta_{2, n+1}=\theta_{2}\right)$. They respectively correspond to the 4 blue wavy lines in figure 12. The other propagators on $\Sigma_{n}$ can not contribute to $2 n$ point functions. Here we assume that an operator $\phi$ is located at $\left(t=-t_{1}, x^{1}=-l\right)\left(t_{1}>l\right)$. In other words, we explain features of propagators in the region where $\Delta S_{A}^{(n)}$ increase nontrivially.

Even if we take the limit $\epsilon \rightarrow 0$ and $t \rightarrow \infty$, those dominant propagators can contribute to $\Delta S_{A}^{(n)}$. The ratio of $D^{(n)}$ to $D^{(1)}$ does not depend on the number of sheets and the spacetime dimension. It is given by

$$
\frac{D^{(n)}}{D^{(1)}}=\frac{\text { the number of sheets }}{\text { the number of propagators on the circle }}=\frac{n}{2 n}=\frac{1}{2} .
$$

By using this property of dominant propagators, we can obtain the final value $\Delta S_{A}^{(n) f}$ for : $\phi^{k}$ : in any dimensions. There are two contributions to the $2 n$ point correlation function of : $\phi^{k}:$ on $\Sigma_{n}$. One of them corresponds to the product of propagators between two points on the same sheet. They correspond to red wavy lines in the right figure of figure 12. Another one corresponds to the product of propagators between $\left(r_{1}, \theta_{1, k}\right)$ and $\left(r_{2}, \theta_{2, k+1}\right)$. They correspond to blue wavy lines in the right figure of figure 12 . Then the $2 n$ point function of : $\phi^{k}:$ on $\Sigma_{n}$ is given by

$$
\begin{aligned}
\langle: & \left.\phi^{k}\left(r_{1}, \theta_{1}\right):: \phi^{k}\left(r_{2}, \theta_{2}\right): \cdots: \phi^{k}\left(r_{1}, \theta_{1}+2 \pi(n-1)\right):: \phi^{k}\left(r_{2}, \theta_{2}+2 \pi(n-1)\right):\right\rangle \\
& =(k !)^{n}\left(\frac{D^{(1)}}{2}\right)^{k n}+\left(k !_{k} C_{1}\right)^{n}\left(\frac{D^{(1)}}{2}\right)^{k n}+\cdots+(k !)^{n}\left(\frac{D^{(1)}}{2}\right)^{k n} \\
& =\sum_{l}^{k}\left(k !_{k} C_{l}\right)^{n}\left(\frac{D^{(1)}}{2}\right)^{k n},
\end{aligned}
$$

where we think about the $2 n$ point function of : $\phi^{k}$ : after performing the analytic continuation in $(2.5)$. 
$\left\langle: \phi^{k}:: \phi^{k}:\right\rangle^{n}$ is given by

$$
\left\langle: \phi^{k}:\left(r_{1}, \theta_{1}\right): \phi^{k}:\left(r_{2}, \theta_{2}\right)\right\rangle^{n}=\left(k !\left(D^{(1)}\right)^{k}\right)^{n}
$$

They are proportional to $\left(D^{(1)}\right)^{n k}$ respectively. Then $D^{(1)}$ is cancelled out when we compute $\Delta S_{A}^{(n)}$. Therefore $\Delta S_{A}^{(n) f}$ does not depend on the detail of 2 point function of $\phi$. In any dimensions, $\Delta S_{A}^{(n) f}$ is given by

$$
\Delta S_{A}^{(n) f}=\frac{1}{1-n} \log \left[\frac{1}{2^{n k}} \sum_{l=1}^{k}\left({ }_{k} C_{l}\right)^{n}\right]
$$

In the previous paper [36], we obtained (Rényi) entanglement entropies of : $\phi^{k}:$ under the entangled pair interpretation. However we did not obtain them for any $\mathrm{k}$ and $\mathrm{n}$ by the replica method. In the present paper, we obtain them for any $\mathrm{k}$ and $\mathrm{n}$ by using the distinctive property of propagators. Moreover the result in (5.4) agrees with that which we obtained under the entangled pair interpretation. The ratio of $D^{(n)}$ to $D^{(1)}$ does not depend on the details of the propagators. Therefore, it is expected that that ratio can be generalized to that for propagators of general fields in the free massless scalar field theory.

\subsubsection{Time evolution of (Rényi) entanglement entropies for excited states}

We would like to comment on the time evolution of $\Delta S_{A}^{(n)}$ for the states defined by acting $: \phi^{k}$ : on the ground state. Here we assume that it is located at $\left(t=-t_{1}, x^{1},=-l\right)\left(t_{1}>l\right)$. In 4 dimensions, we are able to find time dependence of dominant propagators. Its time dependence does not depend on the number of sheets in the limit $\epsilon \rightarrow 0$. In the limit $\epsilon \rightarrow 0$, the dominant propagators between two points on the same sheet are given by

$$
D_{s}^{(n)}=\frac{l+t_{1}}{32 \pi^{2} t_{1} \epsilon^{2}}+\mathcal{O}\left(\epsilon^{0}\right)
$$

In this limit, dominant propagators between $\left(r_{1}, \theta_{1, k}\right)$ and $\left(r_{2} \cdot \theta_{2, k+1}\right)$ are given by

$$
D_{a}^{(n)}=\frac{-l+t_{1}}{32 \pi^{2} t_{1} \epsilon^{2}}+\mathcal{O}\left(\epsilon^{0}\right)
$$

We can investigate the time evolution of (Rényi) entanglement entropies for the states excited by $: \phi^{k}:$. Their growth is given by

$$
\Delta S_{A}^{(n)}=\frac{1}{1-n} \log \left[\left(16 \pi^{2} \epsilon^{2}\right)^{k n} \sum_{i=0}^{k}\left({ }_{k} C_{i}\right)^{n}\left(D_{a}^{(n)}\right)^{n i}\left(D_{s}^{(n)}\right)^{n(k-i)}\right] \quad t \geq l
$$

where $\Delta S_{A}^{(n)}$ is finite in the limit $\epsilon \rightarrow 0$. 


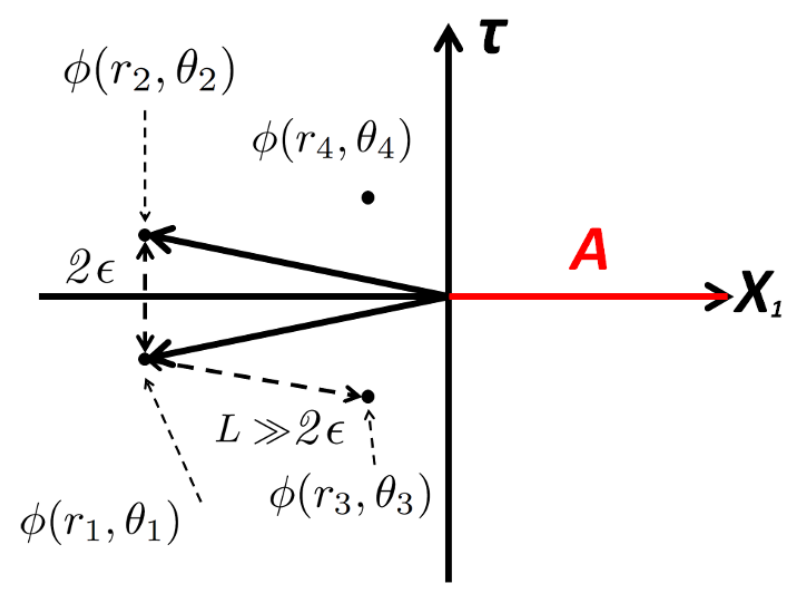

Figure 13. The locations of operators on $\Sigma_{1}$. The distance $L$ between $\phi\left(r_{1}, \theta_{1}\right)$ and $\phi\left(r_{3}, \theta_{3}\right)$ is much larger than the distance $2 \epsilon$ between $\phi\left(r_{1}, \theta_{1}\right)$ and $\phi\left(r_{2}, \theta_{2}\right)$.

\subsection{Sum rule of (Rényi) entanglement entropies of local operators}

By the replica method, we will obtain the sum rule which (Rényi) entanglement entropies obey. Here $p$ means the position of composite operators and $k$ means the powers of $\phi$. We act operators $\mathcal{O}_{p_{1}}^{k_{1}}, \mathcal{O}_{p_{2}}^{k_{2}}, \cdots, \mathcal{O}_{p_{l}}^{k_{l}}$ on the ground state. They are located separately. We can find out final values of $\Delta S_{A}^{(n)}$ in the limit $\epsilon \rightarrow 0$ by the replica method.

In the present case there are also correlations between operators which are located separately. At first, it seems that they can contribute to (Rényi) entanglement entropies for locally excited states after performing the analytic continuation in (2.5). However only dominant propagators can contribute to $\Delta S_{A}^{(n)}$ if we take the limit $\epsilon \rightarrow 0$. In this limit, the leading term of dominant propagators is given by

$$
D^{(n)} \sim \mathcal{O}\left(\left(\epsilon^{-2}\right)^{\frac{d-1}{2}}\right)
$$

where $d>2$.

On the other hand, the leading term of the propagators between operators which are located separately is much smaller than that of dominant propagators. Therefore they can not contribute to $\Delta S_{A}^{(n)}$. We are able to explain why only dominant propagators can contribute to $\Delta S_{A}^{(n)}$ as follows. We assume that two operators $\phi\left(r_{1}, \theta_{1}\right)$ and $\phi\left(r_{3}, \theta_{3}\right)$ are inserted into $\Sigma_{1}$. We define $\phi\left(r_{2}, \theta_{2}\right)$ and $\phi\left(r_{4}, \theta_{4}\right)$ as copies of $\phi\left(r_{1}, \theta_{1}\right)$ and $\phi\left(r_{3}, \theta_{3}\right)$ respectively. We also define $\phi\left(r_{a}, \theta_{a, i}\right)$ as their replica fields on each sheet $i$ respectively $(a=1, \cdots, 4, i=1, \cdots, n)$. On $\Sigma_{1}, \epsilon$ is a regularization parameter for the correlation function of $\phi\left(r_{1}, \theta_{1}\right)$ and its copy $\phi\left(r_{2}, \theta_{2}\right)$ as in figure 13. The distance between $\phi\left(r_{1}, \theta_{1, i}\right)$ and $\phi\left(r_{2}, \theta_{2, j}\right)$ greatly depends on the parameter $\epsilon$. On the other hand, the distance between $\phi\left(r_{1}, \theta_{1, i}\right)$ and $\phi\left(r_{3}, \theta_{3, j}\right)$ or $\phi\left(r_{4}, \theta_{4, j}\right)$ does not depend on $\epsilon$ compared to the distance between $\phi\left(r_{1}, \theta_{1, i}\right)$ and $\phi\left(r_{2}, \theta_{2, j}\right)$. Here $\phi\left(r_{3}, \theta_{3}\right)$ is put on the different point from the location of $\phi\left(r_{1}, \theta_{1}\right)$ on $\Sigma_{1}$. The distance between $\phi\left(r_{1}, \theta_{1, i}\right)$ and $\phi\left(r_{2}, \theta_{2, j}\right)$ shrinks compared to the distance between $\phi\left(r_{1}, \theta_{1, i}\right)$ and $\phi\left(r_{3}, \theta_{3, j}\right)$. Therefore only dominant 
propagators can be greatly enhanced compared to the other propagators when we take the limit $\epsilon \rightarrow 0$. In any dimensions, $\Delta S_{A}^{(n)}$ is given by the sum of (Rényi) entanglement entropies $\Delta S_{A}^{(n), m}$ of each operator,

$$
\Delta S_{A}^{(n)}=\Delta S_{A}^{(n), 1}+\Delta S_{A}^{(n), 2}+\cdots+\Delta S_{A}^{(n), q}=\sum_{i=1}^{q} \Delta S_{A}^{(n), i},
$$

and $\Delta S_{A}^{(n) f}$ also obey the similar relation,

$$
\Delta S_{A}^{(n) f}=\Delta S_{A}^{(n) f, 1}+\Delta S_{A}^{(n) f, 2}+\cdots+\Delta S_{A}^{(n) f, q}=\sum_{i=1}^{q} \Delta S_{A}^{(n) f, i} .
$$

\subsection{Generalization of sum rule}

In previous subsections, we obtained (Rényi) entanglement entropies of $: \phi^{k}$ : and the sum rule which they obey by the replica method. Only dominant propagators can contribute to them and their computation is very simplified in the limit $\epsilon \rightarrow 0$. In the argument about propagators, we do not use a specific property of the propagators of $\phi$.

Therefore we argue that propagators of derivatives of scalar field such as $\partial^{k} \phi$ which are aligned on the circle dominantly contribute to their $2 n$ point correlation functions. We define their dominant propagators on $\Sigma_{n}$ by $G^{(n)}$. They are given by

$$
G^{(n)}=\frac{\text { the number of sheets }}{\text { the number of propagators on a cirle }} G^{(1)}=\frac{n}{2 n} G^{(1)}=\frac{1}{2} G^{(1)} .
$$

If the excited state is defined by acting operators which composed of only single-species operators such as : $\left(\partial^{m} \phi\right)^{k}$ : on the ground state, then the final values of their entropies are given by (5.4) in any dimensions. For example, if the local operator is given by : $\left(\partial_{r} \phi\right)^{k}$ :, then the final value of $n$-th Rényi entanglement entropy is given by

$$
\Delta S_{A}^{(n) f}=\frac{1}{1-n} \log \left[\frac{1}{2^{n k}} \sum_{l=0}^{k}\left({ }_{k} C_{l}\right)^{n}\right] .
$$

We calculate $\Delta S_{A}^{(2,3)}$ for the state defined by acting local operators : $\left(\partial_{r} \phi\right)^{k}$ : on the ground state directly and we check they agrees with the result in (5.12). Furthermore the results which we obtained by the replica method are consistent with that in (5.12).

In turn we prepare an excited state defined by acting various operators on the ground state such as

$$
|\psi\rangle=\mathcal{N}^{-1} \mathcal{T} \prod_{i}^{k} \mathcal{O}^{i}\left(t^{i}, x^{1, i}\right)|0\rangle
$$

where $\mathcal{O}^{i}$ are general local operators and they are located separately. The index $i$ denotes their location.

In this case, it seems that the correlation between $\mathcal{O}^{a}\left(t^{a}, x^{1, a}\right)$ and $\mathcal{O}^{b}\left(t^{b}, x^{1, b}\right)(a \neq b)$ can contribute to $\Delta S_{A}^{(n)}$. However their magnitudes are also much smaller than those of 
dominant propagators when we take the limit $\epsilon \rightarrow 0$. Therefore we argue that in any dimensions, (Rényi) entanglement entropies for the state in (5.13) should obey

$$
\Delta S_{A}^{(n)}=\sum_{m}^{q} \Delta S_{A}^{(n), m},
$$

where $\Delta S_{A}^{(n), k}$ are entropies for the state excited by $\mathcal{O}^{k}$. Their final values of those entropy also obey the similar rule to that in (5.14). They are given by the sum of entropies of $\mathcal{O}^{i}$.

\subsection{Conical singularity}

We construct an $n$ sheeted geometry by gluing the subsystem $\mathrm{A}$ on a sheet to the subsystem A on the next sheet as in figure 2. There is a conical singularity on the boundary $\partial A$ of the subsystem A on the $n$ sheeted geometry. In our case, it is located at $\left(\tau=0, x^{1}=0\right)$. If we think about a conformal field theory on the $n$ sheeted in more than 2 dimensions, we need a conformal mass term $c_{1} \int d x^{d+1} \mathcal{R} \phi^{2}$. Then the action in that theory is given by

$$
S_{\text {Def }}=\int d x^{d+1} \frac{1}{2} \partial_{\mu} \phi \partial^{\mu} \phi+c_{1} \mathcal{R} \phi^{2},
$$

where $c_{1}$ is a some constant. This conformal mass can make an effect on (Rényi) entanglement entropies if a state is excited. ${ }^{11}$ Therefore we have to take into account its effect. We treat it as an operator. If we take in account the effect of the conical singularity, the $2 n$ point correlation function of an operator $\mathcal{O}$ on $\Sigma_{n}$ is given by

$$
\begin{aligned}
\left\langle\mathcal{O}^{2 n}\right\rangle_{\Sigma_{n}^{\text {con }}} & =\left\langle\mathcal{O}^{2 n}: e^{c_{1} \int d x^{d+1} \mathcal{R} \phi^{2}}:\right\rangle_{\Sigma_{n}} \\
& =\sum_{k=0}^{\infty}\left\langle\mathcal{O}^{2 n}: \frac{\left(c_{1} \int d x^{d+1} \mathcal{R} \phi^{2}\right)^{k}}{k !}:\right\rangle_{\Sigma_{n}} \\
& =\left\langle\mathcal{O}^{2 n}\right\rangle_{\Sigma_{n}}+\sum_{k=1}^{\infty}\left\langle\mathcal{O}^{2 n}: \frac{\left(c_{1} \int d x^{d+1} \mathcal{R} \phi^{2}\right)^{k}}{k !}:\right\rangle_{\Sigma_{n}} .
\end{aligned}
$$

The second term of the last line in (5.16) comes from the conical singularity. In this term, some of $2 n$ operators has to contract with operators at $\left(\tau=0, x^{1}=0\right)$. Propagators between the operator at origin and operators at other point weakly depend on $\epsilon$ compared to dominant propagators. Therefore when we take the limit $\epsilon \rightarrow 0$, the second term in (5.16) can not contribute to the leading term of the $2 n$ point correlation function of $\mathcal{O}$. We can ignore the effect of the conical singularity on the boundary of the region A.

By using a simplest example, we explain why the conical singularity can not contribute to the $2 n$ point function of $\mathcal{O}$. We compute second Rényi entanglement entropy for $\mathcal{N}^{-1} \phi|0\rangle$. The 4 point correlation function of $\phi$ on $\Sigma_{2}$ is given by

$$
\begin{aligned}
\left\langle\phi\left(r_{1}, \theta_{1}\right) \phi\left(r_{2}, \theta_{2}\right) \phi\left(r_{1}, \theta_{1}+2 \pi\right) \phi\left(r_{2}, \theta_{2}+2 \pi\right)\right\rangle_{\Sigma_{2}^{\text {con }}} & \\
= & \left\langle\phi\left(r_{1}, \theta_{1}\right) \phi\left(r_{2}, \theta_{2}\right) \phi\left(r_{1}, \theta_{1}+2 \pi\right) \phi\left(r_{2}, \theta_{2}+2 \pi\right)\right\rangle_{\Sigma_{2}} \\
& +\sum_{k=1}^{\infty}\left\langle\mathcal{O}^{2 n}: \frac{\left(c_{2} \int_{\partial A} d \mathbf{x}^{d-1} \phi^{2}(0,0, \mathbf{x})\right)^{k}}{k !}:\right\rangle_{\Sigma_{n}} .
\end{aligned}
$$

\footnotetext{
${ }^{11}$ The authors in [73] pointed out this effect.
} 
$\left\langle\phi\left(r_{1}, \theta_{1}, \mathbf{x}_{1}\right) \phi\left(0, \theta_{2}, \mathbf{x}_{2}\right)\right\rangle$ are given by

$$
\left\langle\phi\left(r_{1}, \theta_{1}, \mathbf{x}_{1}\right) \phi\left(0, \theta_{2}, \mathbf{x}_{2}\right)\right\rangle=\frac{1}{4 \pi^{2}\left(r_{1}^{2}+\left|\mathbf{x}_{1}-\mathbf{x}_{2}\right|^{2}\right)} .
$$

Its contribution is at most given by

$$
\left\langle\phi\left(r_{1}, \theta_{1}, \mathbf{x}_{1}\right) \phi\left(0, \theta_{2}, \mathbf{x}_{2}\right)\right\rangle \sim \frac{1}{4 \pi^{2} r_{1}^{2}} .
$$

After performing the analytic continuation, its contribution is at most $\mathcal{O}(1)$ in the limit $\epsilon \rightarrow 0$ and $\int_{\partial A} d \mathbf{x}^{d-1}$ is regularized. Therefore the leading term of $\left\langle\phi^{4}\right\rangle_{\Sigma_{2}}$ is $\mathcal{O}\left(\epsilon^{-4}\right)$ and the second term in (5.17) is at most $\mathcal{O}\left(\epsilon^{-2}\right)$ in this limit. Then it can not contribute to the 4 point correlation function of $\phi$. Therefore we can ignore the effect of the conical singularity.

\section{Discussions and conclusions}

In the present paper, we have studied (Rényi) entanglement entropies for excited states defined by acting various local operators on the ground state. We have investigated their time evolution.

Furthermore we have defined the excess of (Rényi) entanglement entropies for those locally excited states. We chose a half $\left(x^{1} \geq 0\right)$ of the total space as the subsystem $\mathrm{A}$ in the present case. Those entropies are related to correlation functions of local operators which act on the ground state. In general, it is difficult to compute (Rényi) entanglement entropies in interacting field theories. For their excesses, all that we have to do is to calculate correlation functions of operators if we compute them perturbatively. It is expected that this will be perturbatively computable in interacting field theories.

By the replica method we computed (Rényi) entanglement entropies for excited states defined by acting specific operators on the ground state in the free massless scalar field theory in even dimensions. We performed an analytic continuation to real time and investigated their time evolution. Those entropies approach finite constants at late time. We found their time evolution can be interpreted in terms of the relativistic propagation of entangled pairs.

We gave explanations of their final values in terms of the entangled pair interpretation. We defined (Rényi) entanglement entropies of operators by their final values. They characterize operators from the viewpoint of quantum entanglement and they are independent of the conformal dimensions of operators. (Rényi) entanglement entropies of $: \phi^{k}:$ are given by (4.11). They do not depend on the spacetime dimension. They do not change if we deform the shape of the subsystem A continuously. They can be defined in any dimensions.

We obtained new results that we summarize below. In the previous paper [36], we computed the n-th Rényi entanglement entropies for the states which are excited by $: \phi^{k}:$ by the replica method when $n, k$ is small. In this paper, we obtained the explanation which shows that (Rényi) entanglement entropies of: $\phi^{k}$ : are given by those of the binomial distribution by the replica trick for any $n$ and $k$ and any dimensions. We checked that they agree with the results which we obtained in terms of the entangled pair. We argued (Rényi) entanglement entropies of specific operators are also given by those of the binomial distribution. 


\begin{tabular}{|c|c|c|}
\hline & Dimension of Spacetime & Entanglement of Operators \\
\hline \multirow{3}{*}{ Second Rényi Entropy } & 4 & $\log \left[\frac{100}{37}\right]$ \\
\cline { 2 - 3 } & 6 & $\log \left[\frac{224}{121}\right]$ \\
\cline { 2 - 3 } & 8 & $\log \left[\frac{676}{253}\right]$ \\
\hline
\end{tabular}

Table 2. Second (Rényi) entanglement entropy of : $\phi \partial_{r} \phi:$ in the limit $\epsilon \rightarrow 0$.

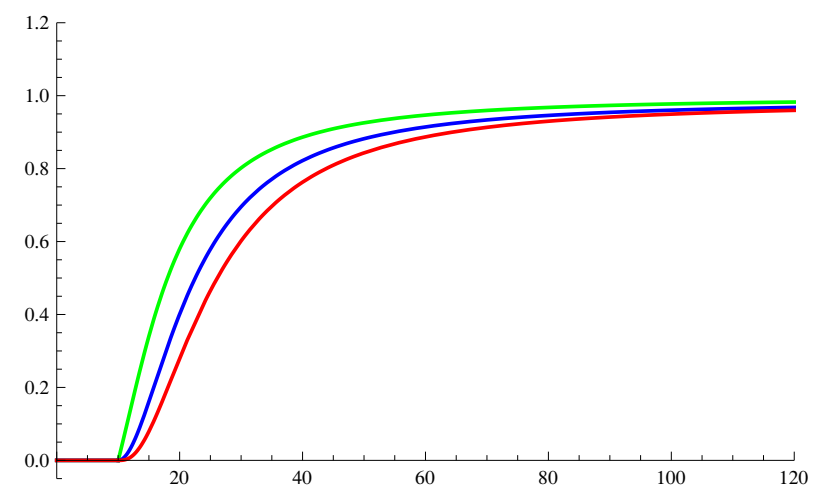

Figure 14. The evolution of $\Delta S_{A}^{(2)}$ with $t_{1}$ in the limit $\epsilon \rightarrow 0$. The vertical axis corresponds to $\Delta S_{A}^{(2)}$. The horizontal axis corresponds to $t_{1}$. Here we chose $l=10$. The green curve is the time evolution of $\Delta S_{A}^{(2)}$ for $\phi \partial_{r} \phi|0\rangle$ in 4 dimensions. The blue curve is the time evolution of $\Delta S_{A}^{(2)}$ for that state in 6 dimensions. The red curve is the time evolution of $\Delta S_{A}^{(2)}$ for that state in 8 dimensions.

These operators are composed of single-species operators such as : $\left(\partial^{m} \phi\right)^{n}$ : and we call them S-operators. We have studied (Rényi) entanglement entropies of $: \phi^{k}:$ in large $k$ limit.

We also obtained the sum rule for the final value of (Rényi) entanglement entropies for locally excited states in any dimensions. Those excited states were defined by acting various local operators on the ground state. We assumed they were separated and they were given by : $\phi^{k}$ :. We checked that the sum rule which we obtained by the replica method agrees with the one derived from the entangled pair. We argued that $\Delta S_{A}^{(n) f}$ for the states defined by acting general operators on the ground state obey the same sum rule in any dimensions. Here we assumed that those operators were located separately.

We would also like to list a few of open problems. As we mentioned above, we found the evidence that (Rényi) entanglement entropies of S-operators will be given by (4.11). Let us compute (Rényi) entanglement entropies of the operators which are composed of multi-species operators such as : $\phi \partial \phi:,: \phi \partial \partial \phi:$ and so on. They are not S-operators. We call them M-operators. It is expected that (Rényi) entanglement entropies of M-operators will not obey the formula given in (4.11). For example, we prepare the state defined by acting an M-operator : $\phi \partial_{r} \phi$ : on the ground state,

$$
|\Psi\rangle=\mathcal{N}^{-1}: \phi \partial_{r} \phi:|0\rangle .
$$

(Rényi) entanglement entropy of : $\phi \partial_{r} \phi:$ depends on the spacetime dimension as in table 2 . The time evolution of $\Delta S_{A}^{(2)}$ for this state is plotted in figure 14. Second Rényi entanglement entropy does not obey the formula in (4.11). 
Let us interpret it in terms of entangled pairs. We can decompose operators to the left moving modes and the right moving modes respectively,

$$
\begin{aligned}
\phi & =\phi_{L}+\phi_{R}, \\
\partial_{r} \phi & =\partial_{r} \phi_{L}+\partial_{r} \phi_{R} .
\end{aligned}
$$

In this case, the relation between $\phi$ and $\partial_{r} \phi$ is not clear. Then those operators are probably not independent of each other. As we explained, second Rényi entanglement entropy of : $\phi \partial_{r} \phi$ : depends on the spacetime dimension and does not obey the formula in (4.11). Therefore, it is interesting to understand why $\Delta S_{A}^{(2)}$ depends on spacetime dimensions and to interpret it physically. It is one of the open problems.

Other interesting open problems include:

- it is interesting to find the formula for (Rényi) entanglement entropies of M-operators

- it is interesting to investigate the time evolution of (Rényi) entanglement entropy for locally excited states in interacting field theories.

- It will be also interesting to investigate (Rényi) entanglement entropies of operators in the massive theory.

Also we would like to refer to [74] for large $N$ CFTs and refer to [72] for rational CFTs in two dimensions.

\section{Acknowledgments}

MN would like to thank the collaborators T. Takayanagi, and T. Numasawa for reading the draft of this paper and giving us very useful and remarkable comments. MN also thanks P. Caputa, S. He and N. Shiba for reading the draft of this paper and giving us useful and remarkable comments. MN is supported by JSPS fellowship.

Open Access. This article is distributed under the terms of the Creative Commons Attribution License (CC-BY 4.0), which permits any use, distribution and reproduction in any medium, provided the original author(s) and source are credited.

\section{References}

[1] M. Levin and X.G. Wen, Detecting Topological Order in a Ground State Wave Function, Phys. Rev. Lett. 96 (2006) 110405 [cond-mat/0510613].

[2] A. Kitaev and J. Preskill, Topological entanglement entropy, Phys. Rev. Lett. 96 (2006) 110404 [hep-th/0510092] [INSPIRE].

[3] B. Hsu, M. Mulligan, E. Fradkin and E.-A. Kim, Universal entanglement entropy in 2D conformal quantum critical points, Phys. Rev. B 79 (2009) 115421 [arXiv:0812.0203] [INSPIRE].

[4] H. Li and F.D.M. Haldane, Entanglement Spectrum as a Generalization of Entanglement Entropy: Identification of Topological Order in Non-Abelian Fractional Quantum Hall Effect States, Phys. Rev. Lett. 101 (2008) 010504 [arXiv:0805.0332]. 
[5] S.T. Flammia, A. Hamma, T.L. Hughes and X.-G. Wen, Topological Entanglement Rényi Entropy and Reduced Density Matrix Structure, Phys. Rev. Lett. 103 (2009) 261601 [arXiv: 0909.3305].

[6] M.A. Metlitski, C.A. Fuertes and S. Sachdev, Entanglement Entropy in the $O(N)$ model, Phys. Rev. B 80 (2009) 115122 [arXiv:0904.4477].

[7] M.B. Hastings, I. Gonzalez, A.B. Kallin and R.G. Melko, Measuring Renyi Entanglement Entropy in Quantum Monte Carlo Simulations, Phys. Rev. Lett. 104 (2010) 157201 [arXiv: 1001.2335$]$.

[8] B. Swingle, Entanglement Renormalization and Holography, Phys. Rev. D 86 (2012) 065007 [arXiv: 0905.1317] [INSPIRE].

[9] B. Swingle, Constructing holographic spacetimes using entanglement renormalization, arXiv:1209.3304 [INSPIRE].

[10] M. Van Raamsdonk, Building up spacetime with quantum entanglement, Gen. Rel. Grav. 42 (2010) 2323 [arXiv: 1005.3035] [InSPIRE].

[11] M. Van Raamsdonk, Comments on quantum gravity and entanglement, arXiv:0907.2939 [INSPIRE].

[12] S. Ryu and T. Takayanagi, Aspects of Holographic Entanglement Entropy, JHEP 08 (2006) 045 [hep-th/0605073] [INSPIRE].

[13] S. Ryu and T. Takayanagi, Holographic derivation of entanglement entropy from AdS/CFT, Phys. Rev. Lett. 96 (2006) 181602 [hep-th/0603001] [INSPIRE].

[14] M. Nozaki, S. Ryu and T. Takayanagi, Holographic Geometry of Entanglement Renormalization in Quantum Field Theories, JHEP 10 (2012) 193 [arXiv:1208.3469] [INSPIRE].

[15] A. Mollabashi, M. Nozaki, S. Ryu and T. Takayanagi, Holographic Geometry of cMERA for Quantum Quenches and Finite Temperature, JHEP 03 (2014) 098 [arXiv:1311.6095] [INSPIRE].

[16] M. Nozaki, T. Numasawa, A. Prudenziati and T. Takayanagi, Dynamics of Entanglement Entropy from Einstein Equation, Phys. Rev. D 88 (2013) 026012 [arXiv:1304.7100] [INSPIRE].

[17] J. Bhattacharya and T. Takayanagi, Entropic Counterpart of Perturbative Einstein Equation, JHEP 10 (2013) 219 [arXiv:1308.3792] [INSPIRE].

[18] T. Faulkner, M. Guica, T. Hartman, R.C. Myers and M. Van Raamsdonk, Gravitation from Entanglement in Holographic CFTs, JHEP 03 (2014) 051 [arXiv: 1312.7856] [INSPIRE].

[19] N. Lashkari, M.B. McDermott and M. Van Raamsdonk, Gravitational dynamics from entanglement 'thermodynamics', JHEP 04 (2014) 195 [arXiv:1308.3716] [INSPIRE].

[20] J. Bhattacharya, M. Nozaki, T. Takayanagi and T. Ugajin, Thermodynamical Property of Entanglement Entropy for Excited States, Phys. Rev. Lett. 110 (2013) 091602 [arXiv: 1212.1164] [INSPIRE].

[21] D.D. Blanco, H. Casini, L.-Y. Hung and R.C. Myers, Relative Entropy and Holography, JHEP 08 (2013) 060 [arXiv: 1305.3182] [INSPIRE].

[22] D. Allahbakhshi, M. Alishahiha and A. Naseh, Entanglement Thermodynamics, JHEP 08 (2013) 102 [arXiv:1305.2728] [INSPIRE]. 
[23] M. Nozaki, T. Numasawa and T. Takayanagi, Holographic Local Quenches and Entanglement Density, JHEP 05 (2013) 080 [arXiv: 1302.5703] [INSPIRE].

[24] W.-z. Guo, S. He and J. Tao, Note on Entanglement Temperature for Low Thermal Excited States in Higher Derivative Gravity, JHEP 08 (2013) 050 [arXiv: 1305.2682] [INSPIRE].

[25] A. Bhattacharyya, A. Kaviraj and A. Sinha, Entanglement entropy in higher derivative holography, JHEP 08 (2013) 012 [arXiv: 1305.6694] [INSPIRE].

[26] S. He, D. Li and J.-B. Wu, Entanglement Temperature in Non-conformal Cases, JHEP 10 (2013) 142 [arXiv:1308.0819] [INSPIRE].

[27] P. Caputa, V. Jejjala and H. Soltanpanahi, Entanglement entropy of extremal BTZ black holes, Phys. Rev. D 89 (2014) 046006 [arXiv:1309.7852] [INSPIRE].

[28] D.-W. Pang, Entanglement thermodynamics for nonconformal D-branes, Phys. Rev. D 88 (2013) 126001 [arXiv:1310.3676] [INSPIRE].

[29] A. Bhattacharyya, M. Sharma and A. Sinha, On generalized gravitational entropy, squashed cones and holography, JHEP 01 (2014) 021 [arXiv:1308.5748] [INSPIRE].

[30] A. Bhattacharyya and M. Sharma, On entanglement entropy functionals in higher derivative gravity theories, arXiv:1405.3511 [INSPIRE].

[31] P. Caputa, G. Mandal and R. Sinha, Dynamical entanglement entropy with angular momentum and $\mathrm{U}(1)$ charge, JHEP 11 (2013) 052 [arXiv: 1306.4974] [INSPIRE].

[32] G. Wong, I. Klich, L.A. Pando Zayas and D. Vaman, Entanglement Temperature and Entanglement Entropy of Excited States, JHEP 12 (2013) 020 [arXiv:1305.3291] [INSPIRE].

[33] J. Cardy, Some results on the mutual information of disjoint regions in higher dimensions, J. Phys. A 46 (2013) 285402 [arXiv:1304.7985] [inSPIRE].

[34] N. Shiba, Entanglement Entropy of Two Black Holes and Entanglement Entropic Force, Phys. Rev. D 83 (2011) 065002 [arXiv:1011.3760] [InSPIRE].

[35] N. Shiba, Entanglement Entropy of Two Spheres, JHEP 07 (2012) 100 [arXiv:1201.4865] [INSPIRE].

[36] M. Nozaki, T. Numasawa and T. Takayanagi, Quantum Entanglement of Local Operators in Conformal Field Theories, Phys. Rev. Lett. 112 (2014) 111602 [arXiv:1401.0539] [InSPIRE].

[37] F.C. Alcaraz, M.I. Berganza and G. Sierra, Entanglement of low-energy excitations in Conformal Field Theory, Phys. Rev. Lett. 106 (2011) 201601 [arXiv:1101.2881] [InSPIRE].

[38] V. Alba, M. Fagotti and P. Calabrese, Entanglement entropy of excited states, J. Stat. Mech. 0910 (2009) P10020 [arXiv:0909.1999] [INSPIRE].

[39] F.C. Alcaraz, M.I. Berganza and G. Sierra, Entanglement of low-energy excitations in Conformal Field Theory, Phys. Rev. Lett. 106 (2011) 201601 [arXiv:1101.2881] [InSPIRE].

[40] P. Calabrese and J.L. Cardy, Evolution of entanglement entropy in one-dimensional systems, J. Stat. Mech. 0504 (2005) P04010 [cond-mat/0503393] [INSPIRE].

[41] P. Calabrese and J.L. Cardy, Time-dependence of correlation functions following a quantum quench, Phys. Rev. Lett. 96 (2006) 136801 [cond-mat/0601225] [INSPIRE].

[42] P. Calabrese and J.L. Cardy, Quantum Quenches in Extended Systems, J. Stat. Mech. 0706 (2007) P06008 [arXiv:0704.1880] [INSPIRE]. 
[43] S. Sotiriadis and J.L. Cardy, Quantum quench in interacting field theory: A self-consistent approximation, Phys. Rev. B 81 (2010) 134305 [arXiv: 1002.0167] [INSPIRE].

[44] S. Bhattacharyya and S. Minwalla, Weak Field Black Hole Formation in Asymptotically AdS Spacetimes, JHEP 09 (2009) 034 [arXiv:0904.0464] [INSPIRE].

[45] S.R. Das, T. Nishioka and T. Takayanagi, Probe Branes, Time-dependent Couplings and Thermalization in AdS/CFT, JHEP 07 (2010) 071 [arXiv: 1005.3348] [INSPIRE].

[46] H. Ebrahim and M. Headrick, Instantaneous Thermalization in Holographic Plasmas, arXiv: 1010.5443 [INSPIRE].

[47] D. Garfinkle and L.A. Pando Zayas, Rapid Thermalization in Field Theory from Gravitational Collapse, Phys. Rev. D 84 (2011) 066006 [arXiv:1106.2339] [InSPIRE].

[48] P. Basu and S.R. Das, Quantum Quench across a Holographic Critical Point, JHEP 01 (2012) 103 [arXiv:1109.3909] [INSPIRE].

[49] S.R. Das, Holographic Quantum Quench, J. Phys. Conf. Ser. 343 (2012) 012027 [arXiv:1111.7275] [INSPIRE].

[50] E. Caceres and A. Kundu, Holographic Thermalization with Chemical Potential, JHEP 09 (2012) 055 [arXiv: 1205.2354] [INSPIRE].

[51] A. Buchel, L. Lehner and R.C. Myers, Thermal quenches in $N=2^{*}$ plasmas, JHEP 08 (2012) 049 [arXiv:1206.6785] [INSPIRE].

[52] M.J. Bhaseen, J.P. Gauntlett, B.D. Simons, J. Sonner and T. Wiseman, Holographic Superfluids and the Dynamics of Symmetry Breaking, Phys. Rev. Lett. 110 (2013) 015301 [arXiv: 1207.4194] [INSPIRE].

[53] P. Basu, D. Das, S.R. Das and T. Nishioka, Quantum Quench Across a Zero Temperature Holographic Superfluid Transition, JHEP 03 (2013) 146 [arXiv:1211.7076] [INSPIRE].

[54] X. Gao, A.M. Garcia-Garcia, H.B. Zeng and H.-Q. Zhang, Normal modes and time evolution of a holographic superconductor after a quantum quench, JHEP 06 (2014) 019 [arXiv: 1212.1049] [INSPIRE].

[55] W. Baron, D. Galante and M. Schvellinger, Dynamics of holographic thermalization, JHEP 03 (2013) 070 [arXiv:1212.5234] [INSPIRE].

[56] A. Buchel, L. Lehner, R.C. Myers and A. van Niekerk, Quantum quenches of holographic plasmas, JHEP 05 (2013) 067 [arXiv: 1302.2924] [INSPIRE].

[57] T. Takayanagi and T. Ugajin, Measuring Black Hole Formations by Entanglement Entropy via Coarse-Graining, JHEP 11 (2010) 054 [arXiv: 1008.3439] [InSPIRE].

[58] T. Hartman and J. Maldacena, Time Evolution of Entanglement Entropy from Black Hole Interiors, JHEP 05 (2013) 014 [arXiv: 1303.1080] [INSPIRE].

[59] P. Calabrese and J.L. Cardy, Entanglement and correlation functions following a local quench: a conformal field theory approach, J. Stat. Mech. 10 (2007) P10004 [arXiv: 0708.3750].

[60] V. Eisler, F. Igloi and I. Peschel, Evolution of entanglement after a local quench, J. Stat. Mech. 6 (2007) P06005 [cond-mat/0703379].

[61] T. Ugajin, Two dimensional quantum quenches and holography, arXiv:1311.2562 [INSPIRE]. 
[62] M. Ganahl, E. Rabel, F.H.L. Essler and H.G. Evertz, Observing complex bound states in the spin-1/2 Heisenberg XXZ chain using local quantum quenches, Physical Review Letters 108 (2012) 077206 [arXiv:1112.3355].

[63] A. Kleine, C. Kollath, I. McCulloch, T. Giamarchi and U. Schollwoeck, Spin-charge separation in two-component Bose-gases, Phys. Rev. A 77 (2008) 013607 [arXiv: 0706.0709].

[64] A.F. Astaneh and A.E. Mosaffa, Holographic Entanglement Entropy for Excited States in Two Dimensional CFT, JHEP 03 (2013) 135 [arXiv:1301.1495] [INSPIRE].

[65] S. Ryu and T. Takayanagi, Aspects of Holographic Entanglement Entropy, JHEP 08 (2006) 045 [hep-th/0605073] [INSPIRE].

[66] P. Calabrese and J.L. Cardy, Entanglement entropy and conformal field theory, J. Phys. A 42 (2009) 504005 [arXiv:0905.4013] [InSPIRE].

[67] J.L. Cardy, O.A. Castro-Alvaredo and B. Doyon, Form factors of branch-point twist fields in quantum integrable models and entanglement entropy, J. Statist. Phys. 130 (2008) 129 [arXiv:0706.3384] [INSPIRE].

[68] P. Calabrese and J.L. Cardy, Entanglement entropy and quantum field theory, J. Stat. Mech. 0406 (2004) P06002 [hep-th/0405152] [INSPIRE].

[69] S. Deser and R. Jackiw, Classical and Quantum Scattering on a Cone, Commun. Math. Phys. 118 (1988) 495 [inSPIRE].

[70] M.E.X. Guimaraes and B. Linet, Scalar Green's functions in an Euclidean space with a conical-type line singularity, Commun. Math. Phys. 165 (1994) 297 [INSPIRE].

[71] J.S. Dowker, Quantum Field Theory on a Cone, J. Phys. A 10 (1977) 115 [InSPIRE].

[72] S. He, T. Numasawa, T. Takayanagi and K. Watanabe, Quantum Dimension as Entanglement Entropy in 2D CFTs, Phys. Rev. D 90 (2014) 041701 [arXiv:1403.0702] [INSPIRE].

[73] A. Lewkowycz and J. Maldacena, Exact results for the entanglement entropy and the energy radiated by a quark, JHEP 05 (2014) 025 [arXiv:1312.5682] [INSPIRE].

[74] P. Caputa, M. Nozaki and T. Takayanagi, Entanglement of local operators in large- $N$ conformal field theories, PTEP 2014 (2014) 093B06 [arXiv:1405.5946] [INSPIRE]. 